\title{
Serotonergic Facilitation of Forelimb Functional Recovery in Rats with Cervical Spinal Cord Injury
}

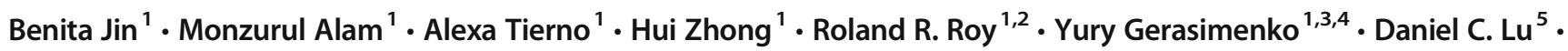 \\ V. Reggie Edgerton ${ }^{1,2,5,6,7,8}$
}

Accepted: 14 November 2020 / Published online: 8 January 2021

(C) The American Society for Experimental NeuroTherapeutics, Inc. 2021

\begin{abstract}
Serotonergic agents can improve the recovery of motor ability after a spinal cord injury. Herein, we compare the effects of buspirone, a $5-\mathrm{HT}_{1 \mathrm{~A}}$ receptor partial agonist, to fluoxetine, a selective serotonin reuptake inhibitor, on forelimb motor function recovery after a $\mathrm{C} 4$ bilateral dorsal funiculi crush in adult female rats. After injury, single pellet reaching performance and forelimb muscle activity decreased in all rats. From 1 to 6 weeks after injury, rats were tested on these tasks with and without buspirone (1-2 $\mathrm{mg} / \mathrm{kg}$ ) or fluoxetine (1-5 mg/kg). Reaching and grasping success rates of buspirone-treated rats improved rapidly within 2 weeks after injury and plateaued over the next 4 weeks of testing. Electromyography (EMG) from selected muscles in the dominant forelimb showed that buspirone-treated animals used new reaching strategies to achieve success after the injury. However, forelimb performance dramatically decreased within 2 weeks of buspirone withdrawal. In contrast, fluoxetine treatment resulted in a more progressive rate of improvement in forelimb performance over 8 weeks after injury. Neither buspirone nor fluoxetine significantly improved quadrupedal locomotion on the horizontal ladder test. The improved accuracy of reaching and grasping, patterns of muscle activity, and increased excitability of spinal motor-evoked potentials after buspirone administration reflect extensive reorganization of connectivity within and between supraspinal and spinal sensory-motor netxcopy works. Thus, both serotonergic drugs, buspirone and fluoxetine, neuromodulated these networks to physiological states that enabled markedly improved forelimb function after cervical spinal cord injury.
\end{abstract}

Key Words Serotonin $\cdot$ Spinal cord injury $\cdot$ Buspirone $\cdot$ Fluoxetine $\cdot$ Forelimb

Benita Jin and Monzurul Alam contributed equally to this work.

Significance Statement Two serotonergic agents-buspirone and fluoxetine - having different mechanisms of action, facilitate forelimb functional recovery after an incomplete cervical lesion. The degree of improvement and the rate of progression differed between the drug treatments. Multiple, novel reaching, and grasping strategies were seen in the forelimb motor activity patterns after buspirone administration after injury. This study highlights the functional potential of serotonergic modulation after cervical cord injury on the forelimb.

\section{Reggie Edgerton}

vre@ucla.edu

1 Department of Integrative Biology and Physiology, University of California, Los Angeles, 610 Charles E. Young Drive, Los Angeles, CA 90095-1527, USA

2 Brain Research Institute, University of California, Los Angeles, Los Angeles, CA 90095, USA

3 Pavlov Institute of Physiology, St. Petersburg 199034, Russia

4 Institute of Fundamental Medicine and Biology, Kazan Federal University, Kazan 420006, Russia
5 Department of Neurosurgery, University of California, Los Angeles, CA 90095, USA

6 Department of Neurobiology, University of California, Los Angeles, CA 90095, USA

7 Faculty of Science, The Centre for Neuroscience and Regenerative Medicine, University of Technology Sydney, Ultimo, NSW, Australia

8 Institut Guttmann, Hospital de Neurorehabilitació, Institut Universitari adscript a la Universitat Autònoma de Barcelona, 08916 Badalona, Spain 


\section{Introduction}

Cervical spinal cord injury (SCI) damages both ascending and descending sensory-motor projections, inducing paralysis and reducing the levels of serotonin (5HT) neurotransmitter in the spinal cord $[1,2]$. Subsequent upregulation of 5HT receptor (5HTR) subtypes 1A, 2A, 2C, and 7 further contributes to the dysregulation of 5HTRs which may be crucial for modulating spinal networks involved in motor tasks [2-4]. Reversal of 5HT loss can pharmacologically improve hindlimb [5] and forelimb [6] recovery in spinalized animals. Here, we sought to determine whether serotonergic drug application after injury can promote forelimb recovery and whether two FDAapproved 5HT agents - selective serotonin receptor inhibitor (SSRI) fluoxetine and $5 \mathrm{HT}_{1 \mathrm{~A}}$ partial receptor agonist buspirone - with distinct mechanisms of action can similarly affect fine motor functions after incomplete cervical SCI.

5HTR agonists and SSRIs can differentially modulate neural networks by targeting a specific set of 5HT receptors to facilitate movement. For example, $5 \mathrm{HT}_{2 \mathrm{~A}} \mathrm{R}$ and $5 \mathrm{HT}_{7} \mathrm{R}$ agonists are sufficient to activate hindlimb spinal cord circuitry $[4$, $7,8]$, and $5 \mathrm{HT}_{1 \mathrm{~A}} \mathrm{R}$ agonists such as buspirone can potentiate coordination during locomotion concomitant with spinal cord stimulation [9]. Besides the spinal cord, $5 \mathrm{HT}_{1 \mathrm{~A}} \mathrm{R}$ agonist 8OH-DPAT can expand forelimb motor maps in the motor cortex [10]. SSRIs can similarly upregulate serotonin by interacting with particular $5 \mathrm{HT}_{2} \mathrm{Rs}$ after neurological injury $[11,12]$. An increase in extracellular $5 \mathrm{HT}$ induced by SSRI fluoxetine was significantly attenuated when $5 \mathrm{HT}_{2 \mathrm{~B}}$ receptors were knocked out genetically or inhibited pharmacologically [13]. While it is clear that both 5HT agents can promote motor function after injury, it is important to note that $5 \mathrm{HTR}$ agonists and SSRIs can have disparate effects. $5 \mathrm{HT}_{1 \mathrm{~A}} \mathrm{R}$ agonists can significantly modulate spatial task behavior, while SSRIs given at a similar dose and route of drug administration does not improve performance despite a similar increase in serotonin levels [14]. Herein, we compare the effects of 5HT1AR and SSRI intervention to determine how the mechanism of 5HT upregulation can differentially affect forelimb dexterity after SCI.

To identify changes in muscle recruitment and coordination during reaching and grasping, we compared electrophysiological recordings of selected forelimb muscles during behavioral tasks and at rest before injury and after 6 weekly postinjury times. Administration of buspirone or fluoxetine after incomplete SCI improved forelimb recovery of reaching and grasping, but not locomotor function by 6 weeks post injury (wpi). Both serotonergic agents improved all components of reaching and grasping over time. These gains were largely lost with buspirone withdrawal and were not regained with buspirone in previously saline-treated rats at 6-8 wpi. Collectively, our results demonstrate that serotonergic modulation can improve forelimb function by enabling different patterns of coordination among motor pools, reflecting functional reorganization of supraspinal-spinal connectomes.

\section{Materials and Methods}

All experimental procedures were approved by the Division of Laboratory Animal Medicine, University of California, Los Angeles, and followed NIH guidelines (National Institutes of Health, Publication No. 86-23, revised 1985).

\section{Animals}

Adult (2-3-month-old) female Long-Evans rats were housed individually in standard Plexiglas cages with a 12-h light/dark cycle. Training and testing were performed during the lighted portion of the cycle. All rats were placed on a food-restricted diet at the start of training of 12-15 g of standard rat chow per day to maintain $90 \%$ of starting body weight. Food was restricted so that rats would not be sated for testing. For the first week after surgery, animals were allowed to have unlimited access to food. After 1 week of unlimited access, post-surgery animals were again maintained on the food-restricted diet. Animals were weighed three times a week to ensure adequate nutrition.

\section{Surgical Implantation of Electrodes}

To further evaluate the use of serotonergic drugs on forelimb recovery, rats were trained on a reaching and grasping task prior to drug treatment. Trained rats were subdivided into two separate study phases, in which the first-phase rats were examined with an incremental dose of serotonergic drugs (buspirone and fluoxetine) while the second-phase rats were examined with a fixed dose of fluoxetine, and examined against two separate control groups (Table 1, see "Drug Administration"). In phase I, five of 20 rats did not learn the single pellet reaching task ( $<60 \%$ success) despite training and were removed from the study. Ten rats from phase I underwent EMG electrode implantation as done in a previous study to record muscle activity patterns concomitant with reaching behavior [15]. Briefly, under aseptic conditions, the rats were deeply anesthetized with $1.5-2.5 \%$ isoflurane gas via a facemask. The skull was exposed, and two multisocket connectors with pre-attached Teflon-coated multistranded stainless steel wires (AS-631, 632; Cooner Wire, Chatsworth, CA) were anchored firmly to the skull via five stainless steel screws and dental cement. The EMG wires were passed subcutaneously from the head connectors to five forelimb muscles (middle deltoid, biceps brachii, pronator teres, flexor digitorum longus, and extensor digitorum longus) in the forelimb that the rat preferred to use in the reaching and grasping task. Two wires then were inserted in the belly of each 
Table 1 Animal subgroups and summary of distribution of rats by experimental group and outcome measures

\begin{tabular}{|c|c|c|c|c|c|}
\hline & \multirow[t]{2}{*}{ Treatment } & \multirow[t]{2}{*}{$N$} & \multicolumn{3}{|l|}{ Outcome measures } \\
\hline & & & Reaching and grasping & Ladder & Electrophysiology \\
\hline \multirow[t]{5}{*}{ Phase I } & \multicolumn{5}{|c|}{ Incremental dosages } \\
\hline & Removed & 5 & $x$ & $x$ & $x$ \\
\hline & Saline & 5 & $\checkmark$ & $\checkmark$ & $\checkmark$ \\
\hline & Buspirone & 5 & $\checkmark$ & $\checkmark$ & $\checkmark$ \\
\hline & Fluoxetine & 5 & $\checkmark$ & $\checkmark$ & $x$ \\
\hline \multirow[t]{4}{*}{ Phase II } & Fixed dosag & & & & \\
\hline & Removed & 6 & $x$ & $x$ & $x$ \\
\hline & Saline & 7 & $\checkmark$ & $\checkmark$ & $x$ \\
\hline & Fluoxetine & 7 & $\checkmark$ & $\checkmark$ & $x$ \\
\hline
\end{tabular}

Forty rats (20 rats per phase) were trained on a single pellet reaching task prior to injury. A total of 11 rats did not learn the task despite training and were removed from the study (i.e., "Removed"). SCI rats treated with incremental dosages of fluoxetine (phase I) did not demonstrate a significant improvement in reaching and grasping performance. At that point, we chose to proceed with the highest dose used in phase I for the phase II (fixed dosage) experiments, focusing on the behavioral results, but not to invest further in the time and cost of the electrophysiological procedures until we obtained results demonstrating its potential as an effective intervention. Thus, all data on fluoxetine in phase I was considered as preliminary. In phase II, the improvement in fine motor skills was compared between saline- and fluoxetine-treated rats muscle, a 1.5-2.0 notch made in the Teflon coating of each wire to make the electrodes, and secured as described in Roy et al. [16]. The electrode wires for spinal motor-evoked potentials (sMEPs) were passed to C6 and C8 spinal cord levels and sutured to dura. The incisions were closed with Vicryl sutures. After surgery, the rats received subcutaneous injections of buprenorphine $(0.05 \mathrm{mg} / \mathrm{kg})$ for pain relief before recovering from anesthesia and every $12 \mathrm{~h}$ for 3 days after surgery, together with $2.5 \mathrm{mg} / \mathrm{kg}$ antibiotic Baytril (Norbrook Laboratories, Lenexa, KS) and $0.6 \mathrm{ml}$ of lactated Ringer's, subcutaneously. The rats recovered in an incubator at $\sim 32{ }^{\circ} \mathrm{C}$ until it recovered from anesthesia and then returned to their home cages. Recording sessions began 7 days postoperatively.

\section{Spinal Cord Injury}

Trained rats from phase I and phase II experiments received a bilateral dorsal funiculi crush (DFC) at cervical level C4. Ten phase I rats received EMG and epidural stimulation electrode implantation (see "Surgical Implantation of Electrodes"), and the DFC was performed 1 month after the implantation. Five phase I rats received the DFC following training. In phase II, six of 20 rats did not learn the single pellet reaching task $(<$ $60 \%$ success) despite training and were removed from the study (Table 1). The remaining fourteen rats from phase II underwent DFC following baseline testing to evaluate the use of fixed doses of fluoxetine on forelimb recovery. Under anesthesia as described above, a dorsal midline incision was made between the occipital bone and the dorsal edge of the T2 vertebra. The underlying muscles were retracted, and a partial laminectomy was performed at the $\mathrm{C} 3$ vertebrae. An incomplete injury of the spinal cord was induced by inserting the tips of Dumont \#2 tweezers (World Precision Instruments, Sarasota, FL) so that each tip was $1 \mathrm{~mm}$ lateral of the midline and $2 \mathrm{~mm}$ deep into the spinal cord parenchyma, spanning the gap between the dorsal root entries $(1.5 \mathrm{~mm}$ lateral to the midline) and keeping them closed for $20 \mathrm{~s}$ [17]. This procedure results in an effective and consistent bilateral sectioning of the dorsal column fibers, ablating the descending dorsal corticospinal tract and ascending fasciculus gracilis and fasciculus cuneatus pathways. This lesion effectively diminishes control of skilled movements in the forelimb. The percentage of lesioned area was not significantly different among saline-, buspirone-, and fluoxetine-treated rats (Fig. 1). Post-surgical care was the same as that described after the implantation surgery. Recording sessions began 7 days postoperatively after injury.

\section{Stimulation Parameters}

sMEPs were collected from EMG-implanted rats for one 30min session/week before and after injury. Each sMEP session was performed after all behavior testing for the week. Monophasic and bipolar epidural stimulation $(2 \mathrm{~Hz})$ at spinal cord levels $\mathrm{C} 6$ and $\mathrm{C} 8$ was used to evoke potentials in selected forelimb muscles of the preferred forelimb at different current intensities using a constant current stimulator (Grass SIU5; Grass Instruments, Warwick, RI) when the rats were awake and at rest. Throughout the study, epidural stimulation of the spinal cord was applied solely for monitoring SMEPs and 

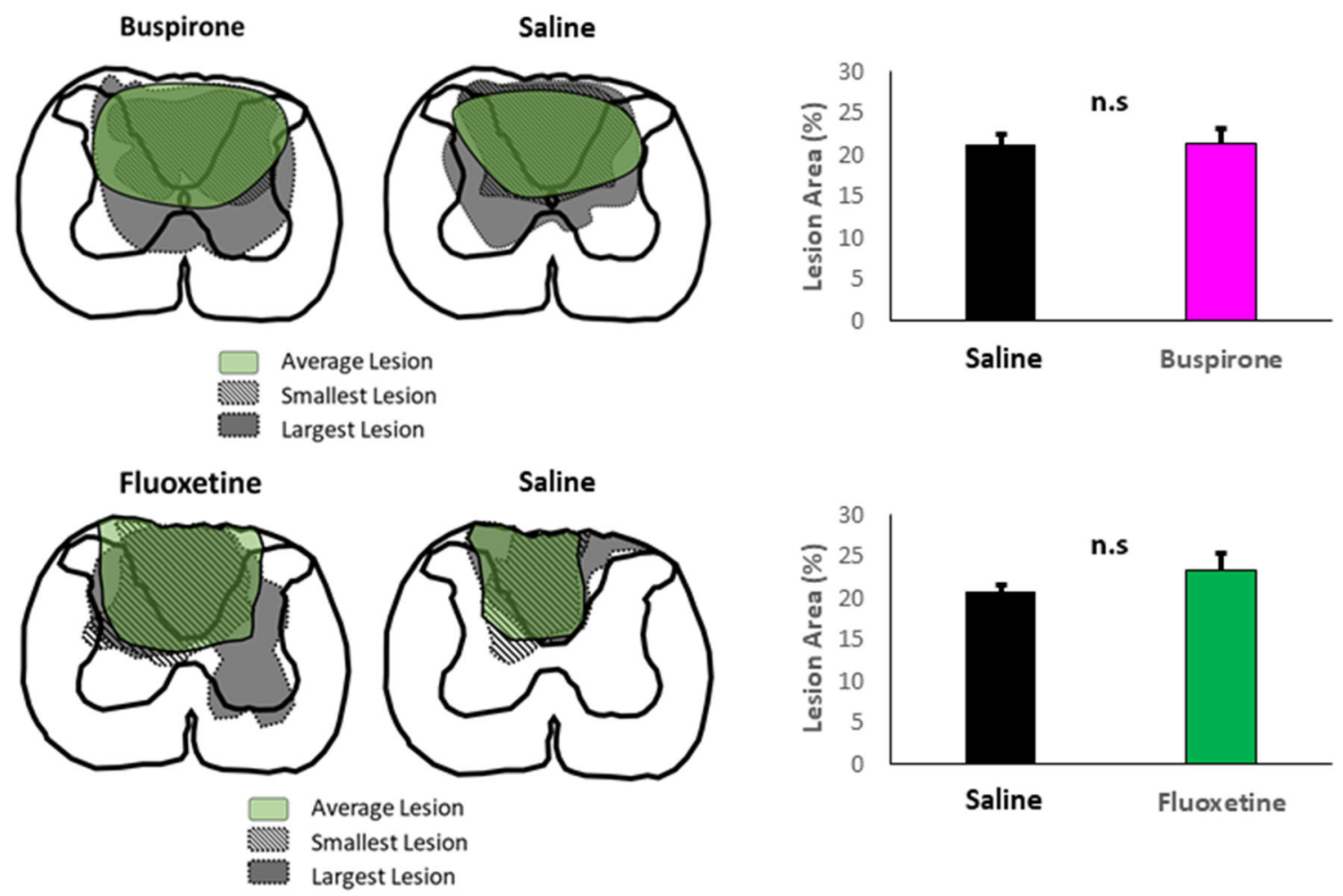

Fig. 1 SCI lesion. Transverse sections $(30 \mu \mathrm{m})$ underwent Nissl staining and imaging and the lesions were outlined using ImagePro and projected onto a heatmap to normalize for individual discrepancies in spinal cord size and width. The percentage of lesion area was calculated as the percentage of the maximum transverse area of the spinal cord at segment $\mathrm{C} 4$.

performed only once per week to avoid a stimulation effect on forelimb recovery.

\section{Drug Administration}

Buspirone dose testing on trained animals from a previous study [18] and fluoxetine administration prior to injury [6] demonstrated the potential of buspirone (Tocris, Bristol, UK) and fluoxetine (Sigma-Aldrich, St. Louis, MO) in facilitating arm and paw movement after a $\mathrm{C} 4$ bilateral dorsal funiculi crush injury. We show here two sets of experiments (phases I and II) that compare the effects of an increasing buspirone dose and a fixed fluoxetine dose on forelimb recovery after SCI (Table 1). Phase I trained rats were divided into three treatment groups with gradually increasing doses from 1 to 6 wpi: saline $(n=5)$, buspirone $(n=5)$, and fluoxetine $(n=$ 5 ). Incremental fluoxetine treatment was given at $1 \mathrm{mg} / \mathrm{kg} / \mathrm{day}$ every week after injury until a maximum dosage of $5 \mathrm{mg} / \mathrm{kg} /$ day was reached at 5 wpi to five rats (Table 1, see "Surgical Implantation of Electrodes"). We subsequently excluded the five rats that were initially assigned to the incremental dosage of fluoxetine in phase I experiments at 6-8 wpi because none of the doses for any given week showed an effective behavioral response to fluoxetine treatment. The remaining 10 rats from phase I ( $n=5$ per group) received a gradual increase in
Representation of the largest, smallest, and average SCI lesions at cervical segment $\mathrm{C} 4$ for buspirone-treated $(n=5)$ and saline-treated $(n=5)$ rats from phase I and for fluoxetine-treated $(n=7)$ and saline-treated $(n=7)$ rats from phase II. Average lesion sizes were not significantly different among groups.

buspirone administration (7 days/week for 8 weeks): 1) buspirone (from 1 to $2 \mathrm{mg} / \mathrm{kg} /$ day) and 2) saline as control (from 1 to $2 \mathrm{mg} / \mathrm{kg} /$ day). Buspirone dosage was increased by $0.5 \mathrm{mg} / \mathrm{kg}$ at 3 wpi and $5 \mathrm{wpi}$ (Fig. 2). These doses were based on previous studies [19-22].

Given that we observed no effect of a gradually increasing dosage of fluoxetine on reaching and grasping in phase I, we performed a second experiment (phase II) using a single fixed dosage (the highest fluoxetine dose used in phase I) from 1 to 6 wpi (Fig. 2). Thus, $n=14$ rats were randomly divided into two treatment groups ( $n=7 /$ group) with fixed drug administration ( 7 days/week for 8 weeks): 1) fluoxetine $(5 \mathrm{mg} / \mathrm{kg} /$ day $)$ and 2) saline as control $(5 \mathrm{mg} / \mathrm{kg} /$ day). Both buspirone and fluoxetine were dissolved in sterile water $(1 \mathrm{mg} / \mathrm{ml})$, sterile filtered, and administered via intraperitoneal injection; $0.9 \%$ isotonic saline (Hanna Pharmaceutical Supply Co., Inc., St. Wilmington, DE) was used as control treatment and given at the same volume as fluoxetine in each phase via intraperitoneal injection. The rats received injections of the same solution every $24 \mathrm{~h}$ for the duration of the experiments. Neither buspirone nor fluoxetine displayed any safety concerns; no tremors or locomotor disturbances were apparent in any of the rats at the dosages used. Recording sessions of reaching and grasping began $30 \mathrm{~min}$ after administration of the drug. 


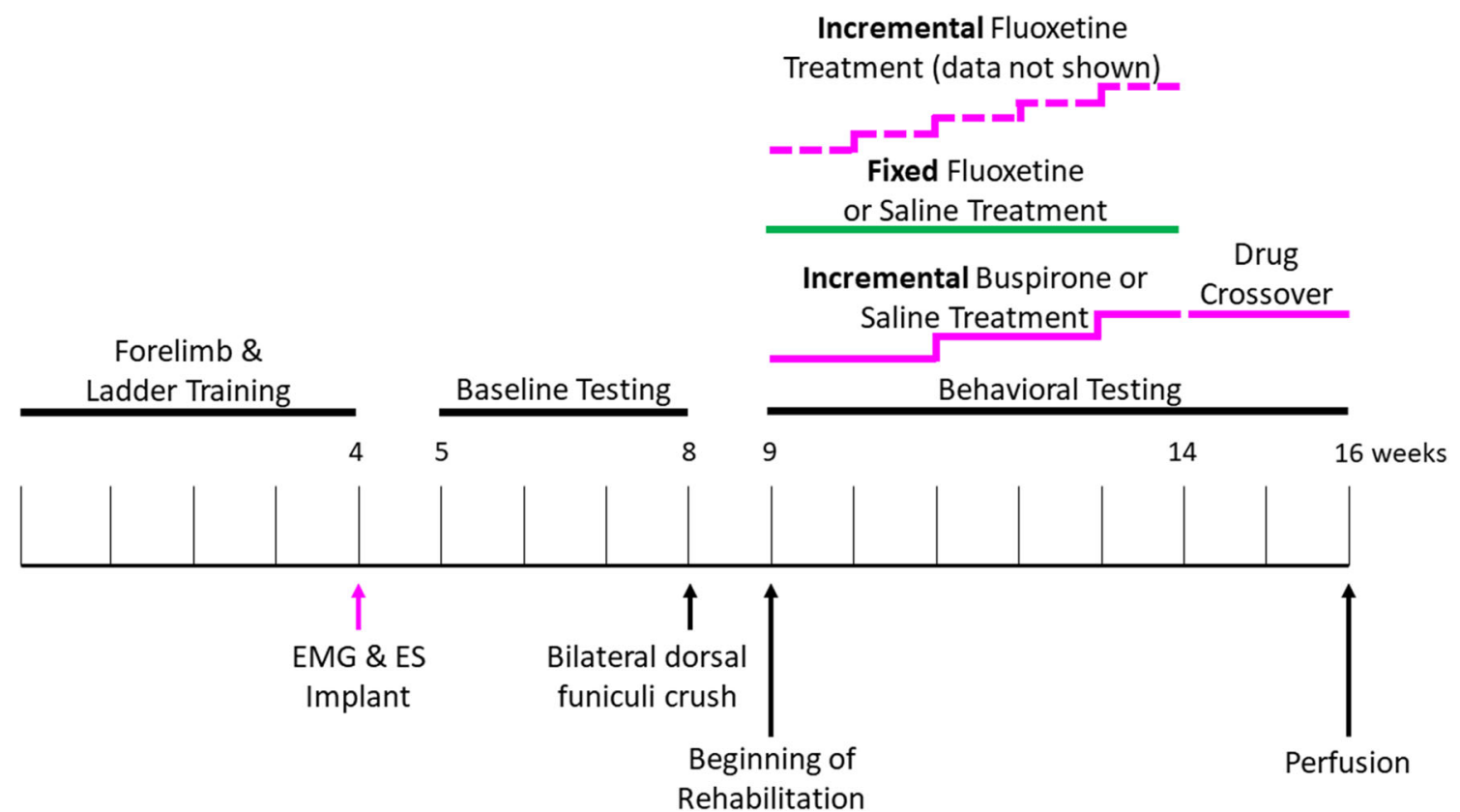

Fig. 2 Experimental design. Drug treatment (magenta, phase I rats; green, phase II rats; black, all rats) was given at 1-8 wpi. Any change in drug dosage across time is shown as a step-wise increment.

\section{Behavioral Assessments}

\section{Reaching and Grasping Task}

Prior to any surgery, all rats were trained on a single pellet reaching and grasping task in a manner similar to that reported by Whishaw et al. [23], Piecharka et al. [24], and García-Alías et al. [25]. The rats were placed in a clear Plexiglas chamber $(15 \mathrm{~cm} \times 30 \mathrm{~cm} \times 17.5 \mathrm{~cm})$ with a narrow window leading to a platform that had two small indentations for the placement of food pellets (banana-flavored, sucrose $45 \mathrm{mg}$ dustless precision pellets; Bio-Serv, Frenchtown, NJ). The rats were trained to reach through the window, grasp the pellet, and retrieve the pellet to its mouth for a period of $10 \mathrm{~min}$ or until they achieved a maximum of 20 attempts per session. The indentations in the platform were positioned so that the rats could not reach the pellets with their tongue and therefore were forced to grasp them with their paw [26]. For each rat, the pellets were placed in the indentation optimal to the rat's natural preferred food position and limb as determined during the first week of training. More than $60 \%$ of the reaching attempts had to be executed with the same forelimb to designate it as the preferred forelimb. Following habituation to the chamber and the reaching and grasping task, the rats were encouraged to move from the front to the back of the chamber after each trial by dropping a pellet in the back of the chamber. This maneuver creates a natural separation between trials and encourages consistency in how the rat orients and initiates itself to perform the next reach [23]. Daily training was stopped after SCI surgery, until the end of the study. Thereafter, the rats were tested on the reaching and grasping task $1 \times /$ week. The testing session was for a maximum of $10 \mathrm{~min}$ and finished when the rat completed 20 attempts. A successful trial for the reaching task was scored when the rat was able to reach, grasp, and eat the pellet. Cases in which the rat made an error in reaching, grasping, or eating were scored as failures. In cases when the rat made multiple attempts sequentially, only the first attempt was scored as a trial. The success rate was calculated by counting the number of successful trials per session and expressed as a percentage of total trials. All success rates were normalized to pre-injury scores. Each testing session was videotaped at 100 frames/s with three video cameras (SIMI Reality Motion Systems, Unterschleissheim, Germany) from the front, left, and right of the rat. A frame grabber was used to capture individual fields for analysis.

\section{Horizontal Ladder Task}

The rats were trained to perform a ladder rung walking task [27] on a horizontal ladder a week prior to surgery. The ladder (1 m long) consisted of side walls made of clear Plexiglas and fitted metal rungs ( $3 \mathrm{~mm}$ diameter) that could be irregularly spaced at distances ranging from 1 to $3 \mathrm{~cm}$. The entire apparatus was elevated $30 \mathrm{~cm}$ above the ground with a neutral start cage and refuge (home cage) at the end. The rats were trained prior to injury with all rungs spaced $1 \mathrm{~cm}$ from each other. After injury, two templates of irregular rung patterns were alternated every other week during testing. Steps from both forelimbs were categorized as 1) miss, in which the paw was placed in between two rungs and did not make contact with any rungs before falling between the rungs, disturbing body posture and balance; 2) slip, in which the paw initially was 
placed on a rung, then slipped upon weight bearing and fell between the rungs, disturbing body posture and balance; 3 ) partial placement, in which the paw was placed on a rung with either the wrist, digits, heels, or toes; or 4) correct placement, in which the mid-portion of the paw was placed on a rung with full weight support and the digits curved around the rung. Misses and slips were considered as footstep errors. Error rates were calculated by counting the number of footstep errors per category and expressed as the average rate per trial.

\section{Data Analysis}

Before the spinal cord injury, all experimental rats (phase I, $n=15$; phase II, $n=14$; Table 1, Fig. 2) were tested and filmed during the single pellet reaching task and the horizontal ladder walking task for baseline measurements of success rates. After injury, the rats were tested $1 \times /$ week, from 1 to 8 wpi. At $6-$ 8 wpi, during the crossover period in the phase I study, the buspirone-treated rats were switched to saline treatment while the saline-treated rats were switched to a buspirone treatment. Video footage of forelimb reaches of each rat was examined frame-by-frame to match to the stages of the reaching and grasping task. Each reaching and grasping attempt was divided into seven stages and qualitatively scored using a modified movement rating scale [23]. Stages were given scores of 1 if the movement appeared normal and similar to pre-injury, 0.5 if the movement was abnormal but recognizable, and 0 if the movement was absent. Footage was scored by five raters blind to the treatment and averaged regardless of the success of the reaching attempt. Reaching attempts were only scored if the rat started with both paws on the ground. The stages were advance (lifting of the forelimb towards the face and body's midline), digit extension (extension of the forelimb from the face towards the pellet platform), arpeggio (digit extension and opening as the paw hovers over the pellet), grasp (paw touches the pellet and its digits close around the pellet), supination I (the paw is withdrawn and supinates by $0-90^{\circ}$ ), supination II (the paw is supinated by $90-120^{\circ}$ and the pellet is brought towards the mouth), and release (the food is transferred from the paw to the mouth). The reach and grasp periods for EMG analysis were normalized to $1 \mathrm{~s}$ each and defined as stages advance-to-grasp and grasp-to-release, respectively.

Video footage of rats crossing the horizontal ladder was analyzed frame-by-frame to score the number of errors and corrections in stepping for each attempt. Five researchers blind to treatment assignment reviewed the videotapes of reaching and stepping sequences and visually counted the number of consecutive successful reaches or steps at each time point. EMG plots for every trial of each muscle recording were generated by a custom MATLAB script based on synchronization of video and EMG recordings. All EMG signals were bandpass filtered at $30-1000 \mathrm{~Hz}$ and amplified $(1000 \times)$ using a multi-channel analog amplifier (Differential AC amplifier Model 1700; AM-Systems, Inc., Sequim, WA). Signals were digitized at $10 \mathrm{kHz}$ with a custom software developed in LabVIEW (National Instruments, Inc., Austin, TX). Time bins for each reach and grasp period during the single pellet task were normalized to a period of $1 \mathrm{~s}$ by interpolation of the digitized data and a custom formula in Excel based on the synchronization of video and EMG recordings. Statistical analyses of area-under-the-curve values for the reach and grasp periods per time point were performed in RStudio (RStudio, Inc., Boston, MA).

For sMEP analysis, a period of rest was defined as forelimb EMG activity being less than 2 standard deviations of baseline activity. Baseline activity was determined by taking the average of activity $100 \mu$ s prior to a stimulation pulse. sMEPs were divided into early ( $<10 \mathrm{~ms}$ after pulse) and late $(10$ $30 \mathrm{~ms}$ after pulse) responses based on modification of the characteristics of these potentials for the forelimb muscles [15]. The latencies, peak-to-peak (P2P) amplitudes, and area-under-the-curve (AUC) values were further filtered with the Teager-Kaiser energy operator (TKEO) by a custom MATLAB code for the early and late sMEP responses [28]. sMEP variables were normalized to pre-injury values for each rat and then transformed to a logarithmic scale for quantitative analysis in RStudio (RStudio, Inc.).

\section{Histological Assessment}

At the end of functional evaluation, animals were perfused with $4 \%$ paraformaldehyde in $0.1 \mathrm{M}$ phosphate-buffered solution (PBS). After perfusion, cervical spinal cord segments were dissected, post-fixed in $4 \%$ paraformaldehyde overnight at $4{ }^{\circ} \mathrm{C}$, and placed in $30 \%$ sucrose in $0.1 \mathrm{M}$ PBS for 2 days. The spinal cord was frozen with Neg-50 (Thermo Scientific, Hudson, $\mathrm{NH}$ ) and tissue blocks stored at $-80{ }^{\circ} \mathrm{C}$ until ready to cut. Blocks were transversely sectioned at $30 \mu \mathrm{m}$ thickness, slide mounted onto Superfrost Plus slides (Fisher Scientific, Springfield, NJ), and kept at $-20{ }^{\circ} \mathrm{C}$ until use. Spinal cord tissues were stained for Nissl substance using $0.1 \%$ Cresyl violet acetate (Sigma-Aldrich) as done in a previous study [29]. Slides were dehydrated by alcohol series (70-100\%), cleared in xylene, and coverslipped with Permount (Fisher Scientific, Waltham, MA). The spinal cord sections were imaged using a Zeiss AxioPhot microscope and Apogee KX-85 camera (Apogee Instruments, Inc., Roseville, CA). Lesion size was quantified using Image-Pro Plus software (Media Cybernetics, Inc., Bethesda, MD).

\section{Statistical Analysis}

Single pellet reaching and ladder walking success rates, temporal and spatial parameters of EMG patterns over time and across groups, and ladder rung steps by time and treatment 
were evaluated by two-way ANOVA and Tukey's HSD post hoc comparisons (JMP10; SAS Institute, Inc., Cary, NC). All box plots were formatted to show the value of the mean (bold center horizontal line), mean \pm standard error (edge of box), and minimum and maximum values of data range (box plot whiskers) in RStudio (RStudio, Inc.). A significant level of $p$ $<0.05$ was set for all comparisons.

\section{Results}

\section{Buspirone Treatment After SCI Improves Forelimb Reaching and Grasping Function}

Buspirone (1-2 mg/kg/day, i.p.) improved the reaching and grasping accuracy of the treatment group as early as $2 \mathrm{wpi}$ (Fig. $\left.3 \mathrm{~A}, F_{(2,31)}=3.022, p=0.005\right)$. Skilled reaching and
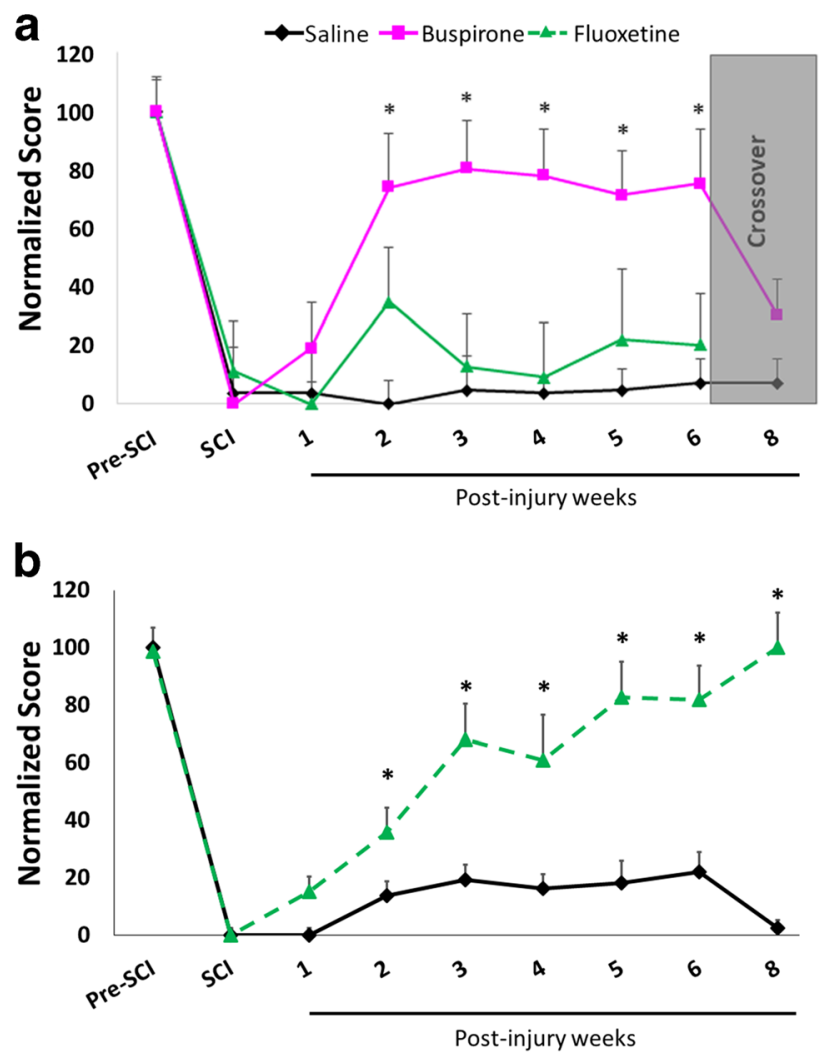

Fig. 3 Reaching and grasping performance of buspirone-treated (magenta, A, C-G) and fluoxetine-treated (green, incremental dosage (A, C-F); fixed dosage $(\mathrm{B}, \mathrm{H}-\mathrm{L}))$ rats versus saline-treated (black) rats over treatment and time. (A, B) Success scores were calculated as percentages of successful reaches among 20 total reaches per time point and normalized to pre-injury scores for each rat. (A) Incremental doses of buspirone $(n=$ 5 ; dose, $1-2 \mathrm{mg} / \mathrm{kg} /$ day), fluoxetine ( $n=5$; dose, $1-5 \mathrm{mg} / \mathrm{kg} /$ day), or saline ( $n=5$; dose, $1-5 \mathrm{mg} / \mathrm{kg} /$ day) were administered beginning 1 wpi. From 6 to $8 \mathrm{wpi}$, rats given buspirone treatment were switched to saline treatment and vice versa (A, crossover). (B) Fixed doses of fluoxetine $(n=7$; dose, $5 \mathrm{mg} / \mathrm{kg} /$ day) or saline $(n=7$; dose, $5 \mathrm{mg} / \mathrm{kg} /$ day $)$ were administered beginning 1 wpi. Each reaching and grasping attempt was divided into seven stages and qualitatively graded per stage (see grasping scores recovered to a slightly lower plateau from 2 to 6 wpi but remained significantly higher than those of salinetreated animals ( 6 wpi, $F_{(2,31)}=2.836, p=0.008$ ). From 6 to 8 wpi, buspirone-treated rats were switched to saline treatment to evaluate the chronic, lasting effects of the serotonin agonist, and the initially saline-treated rats were switched to a buspirone treatment to evaluate the acute effects of the 5HT agonist at a subacute phase of injury (Fig. 3A, gray). After switching the treatments, there was a significant decrease in reaching and grasping accuracy in buspirone-to-saline-treated rats $\left(F_{(2,64)}=2.595, p=0.006\right)$. Reaching and grasping performance did not improve in saline-to-buspirone-treated rats by 8 wpi.

Qualitative analysis of reaching and grasping showed a return of normal movements in buspirone-treated rats and an absence of normal movements in saline-treated rats of phase I, respectively, after injury (Fig. $3 \mathrm{C}-\mathrm{G}$ ). Each reaching and
C

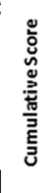

d

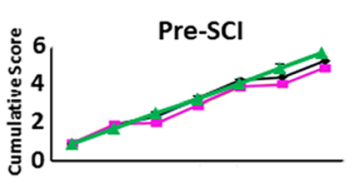

莒

\section{e}

\section{f}

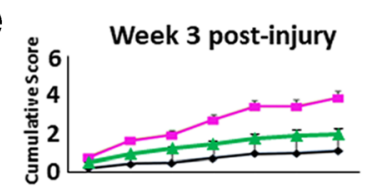

竞,

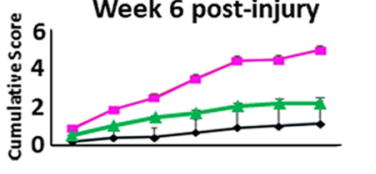

Week 6 post-injury

g

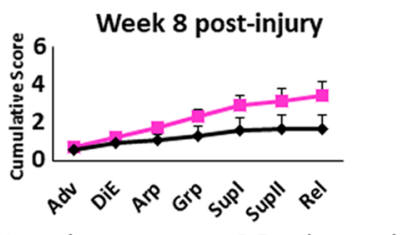

1-Normal movement $\quad 0.5$-abnormal movement

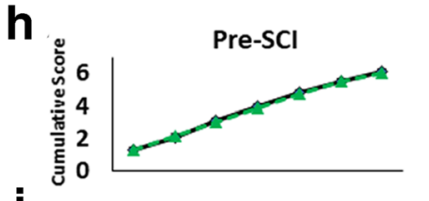

i
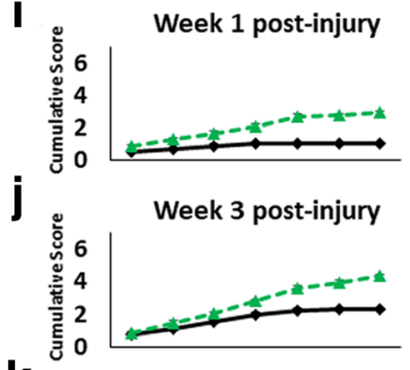

k
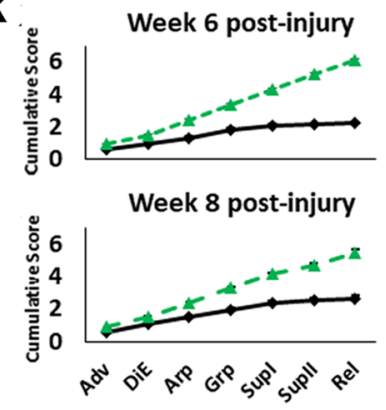

0 -absent movement
"Materials and Methods"). Points were accumulated and averaged according to time point and successful treatment (buspirone $(\mathrm{C}-\mathrm{G})$ and fluoxetine $(\mathrm{H}-\mathrm{L})$ ). Drug-treated rats performed significantly better than saline-treated rats by 3 weeks ((E) total cumulative score at 3 weeks, $F_{(2,341)}=19.545, p<0.0001$, ANOVA; $(\mathrm{F})$ total cumulative score at 6 weeks, $F_{(2,341)}=25.920, p<0.0001$, ANOVA; (I) total cumulative score, $F_{(2,301)}=6.216, p<0.0001$, ANOVA; (J) total cumulative score, $F_{(2,301)}=9.141, p<0.0001$, ANOVA; $(\mathrm{K})$ total cumulative score, $F_{(2,301)}=16.334, p<0.0001$, ANOVA). Error bars indicate SEM. $* p<0.05$, between drug and saline groups. $\mathrm{Adv}=$ advance; $\mathrm{DiE}=$ digit extension; Arp = arpeggio; Grp = grasp; SupI = supination I $\left(0-90^{\circ}\right.$ rotation); SupII = supination II $\left(90-120^{\circ}\right.$ rotation $) ;$ Rel $=$ release . 
grasping attempt was subdivided into seven stages (advance, digit extension, arpeggio, grasp, supination I, supination II, and release) and scored in comparison to each animals' best reaching and grasping trial prior to injury. Scores were sequentially cumulated by stage for each time point. The buspirone-treated rats showed marked improvement in digit extension, grasp, and release stages from 1 wpi compared to saline-treated rats (Fig. 3D, total cumulative score, $F_{(2,341)}=$ $14.857, p<0.0001)$. Saline animals sustained forelimb deficits, particularly during the reaching and grasping periods of the single pellet task, across time (Fig. 3C-F). Buspirone withdrawal partially removed the benefits seen in all stages of the reach, particularly in grasping (Fig. 3G). Regardless of late buspirone intervention at $6 \mathrm{wpi}$, the saline-treated controls' reaching movements minimally improved and were insufficient to rescue forelimb performance (Fig. 3G). Acute, daily administration of buspirone was necessary to improve and maintain skilled forelimb performance after cervical SCI.

\section{Fluoxetine Treatment After SCI Differentially Improves Forelimb Reaching and Grasping Ability}

To determine whether serotonergic drugs with a different functional mechanism than that of buspirone could also facilitate skilled forelimb behavior after injury, we evaluated the administration of SSRI fluoxetine on SCI animals. A gradual dosage of fluoxetine ( $1-5 \mathrm{mg} / \mathrm{kg} /$ day, i.p.) on SCI rats did not significantly improve reaching and grasping despite weekly incremental doses (Fig. 3A, C-F). However, previous studies have shown that fluoxetine dose and application can differentially promote or inhibit neuroplasticity [30,31]. An increased oral dosage of fluoxetine (average of $16.5 \mathrm{mg} / \mathrm{kg} /$ day) prior to an incomplete SCI at cervical level $\mathrm{C} 4$ promoted forelimb recovery after SCI in rats [6]. Prior to the current study, it was unknown whether an increased fluoxetine dose after SCI could facilitate skilled motor function. We performed a second set of experiments comparing SCI rats undergoing saline treatment ( $n=7$, dose of $5 \mathrm{mg} / \mathrm{kg} /$ day, i.p.) or an increased, fixed dosage of fluoxetine $(n=7,5 \mathrm{mg} / \mathrm{kg} /$ day, i.p.) at 1-6 wpi (Fig. 3B). The fixed dosage of fluoxetine significantly improved forelimb function by $2 \mathrm{wpi}\left(F_{(2,126)}=3.197, p=\right.$ $0.002)$. Unlike that of buspirone-treated rats, fluoxetinetreated rats with the higher fixed dosage did not reach a plateau in reaching performance but continued to improve over time. Serotonergic treatment continued to 8 weeks to determine whether fluoxetine-treated rats' scores would eventually plateau below pre-injury scores (Fig. 3B). Fluoxetine-treated rats' scores continued to rise and performed at pre-injury levels $(t(7)=2.466, p=0.022$, one-tailed $t$ test, 6 wpi $v s$ $8 \mathrm{wpi}$ ), while saline-treated rats recovered to a slightly higher plateau before dropping in performance at 8 wpi $(t(7)=2.122$, $p=0.036$, one-tailed $t$ test, 6 wpi vs 8 wpi).
Similar to that of buspirone-treated rats, rats with the higher fixed dosage of fluoxetine demonstrated a return to normal movements in the single pellet task over time (Fig. 3H-L). Both serotonergic agents-buspirone and fluoxetineimproved fine motor movement in the forelimb, particularly during the later phases of the reach and grasp task, while saline treatment did not after cervical SCI.

\section{Electrophysiological Assessment of Forelimb Reaching and Grasping Patterns}

To identify the muscle recruitment strategies used during reaching and grasping, we compared EMG recordings during the single pellet reaching task before and after buspirone treatment. Non-injured animals displayed distinct EMG patterns in all selected forelimb muscles (biceps, deltoid, pronator, extensor digitorum, and flexor digitorum) during the task (Fig. 4A). Alternative strategies include dragging sugar pellets across the pellet platform, and looping above, beyond, and down, and in reverse to retrieve pellets rather than grasping them from above (Movies 1 and 2). Nevertheless, EMG bursts in biceps and deltoid muscles generally correlated to the gross lift of the forelimb, while EMG bursts in the pronator correlated to the rotation of the paw to grasp and retrieval of the pellet to the mouth (Movie 3). The majority of the extensor and flexor muscles' activities was time locked to the reach and grasp periods of the task, respectively, underlining their antagonistic roles during reaching and grasping (Fig. 4A). Ablation of the dorsal corticospinal tract (dCST) severely diminished the duration and magnitude of EMG bursts in all muscles at 1 wpi (Fig. 4B). SCI rats were unable to lift their paws beyond their chins and maintained a slightly clenched paw throughout the task (Movie 4). In the few attempts for which acute SCI rats were able to extend their forelimbs through the window, they overreached laterally beyond the location of the pellet (Fig. 5). Buspirone changed the duration and timing of EMG patterns during the single pellet reaching task from 1 to 2 wpi (Fig. 4C). The amplitude and duration of motor activity in the deltoid and flexor muscles were elevated by buspirone (Movie 5). Buspirone-treated rats continued to overreach at 2 wpi while saline-treated rats returned to placing their paws just in front of or above the pellet, indicating a difference in reaching trajectories (Fig. 5). By 6 wpi, there were distinct differences in EMG patterns between saline- and buspirone-treated rats (Fig. 4D). Saline-treated rats demonstrated low levels of activity in the biceps, deltoid, and flexor muscles; and elevated activity in the pronator muscle. For the few trials that saline-treated rats were able to extend their arms towards the pellet, the biceps activity was dramatically increased for multiple bursts (Movie 6). Moreover, the majority of the extensor activity in saline-treated rats had shifted to the reach period of the task (Fig. 3C). This shift may reflect more wrist extension to stabilize the arm during reaching [32]. Correspondingly, saline-treated rats at 6 wpi, despite the lack of success, on average placed their 


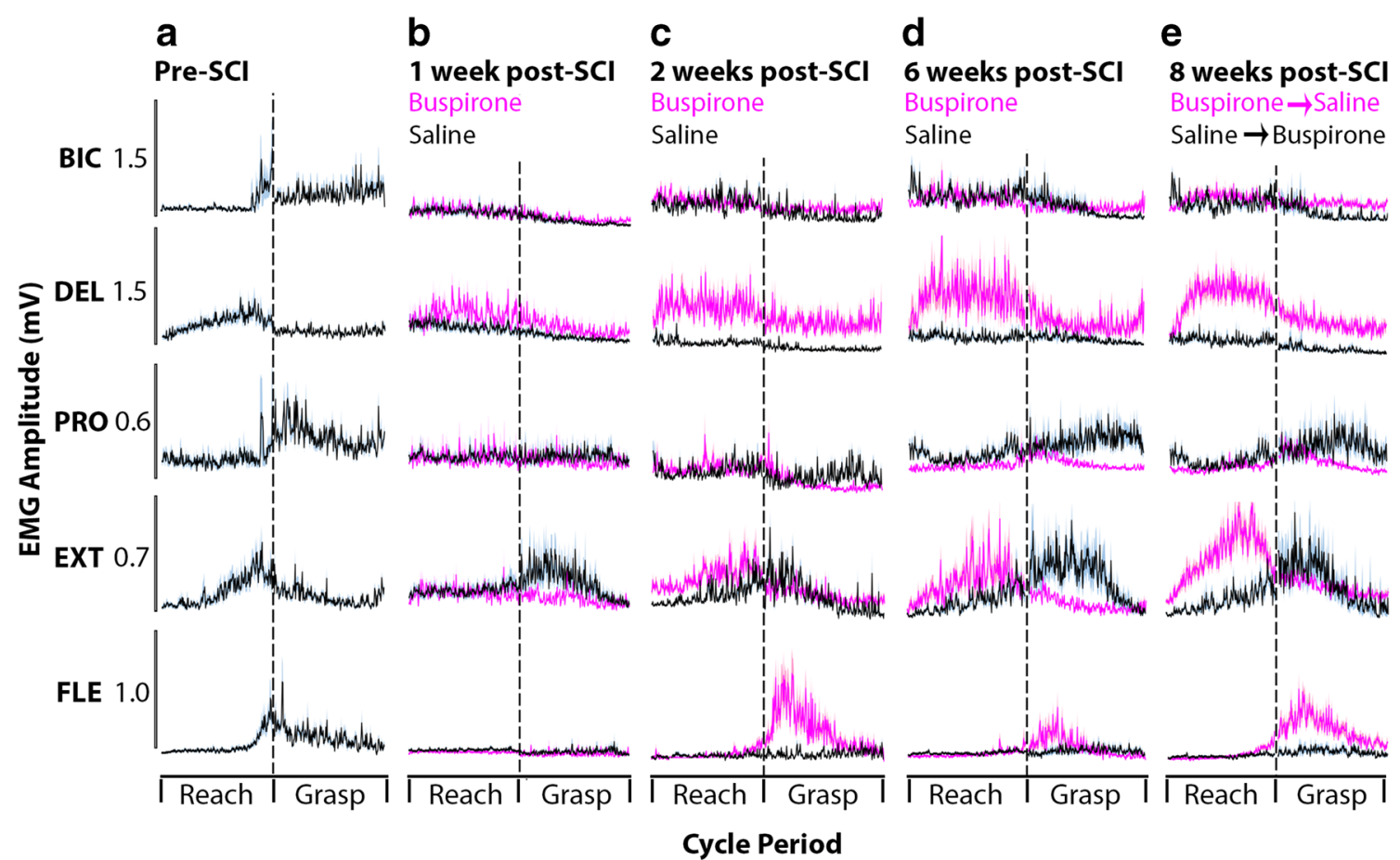

Fig. 4 Rectified normalized EMG activity of selected muscles in the dominant forelimb of four trained rats (buspirone-treated rats, $n=2$; saline-treated rats, $n=2$ ) during the single pellet reaching task (A) before injury ( $n=80$ trials, 4 rats, black) and with ( $n=40$ trials, 2 rats, magenta) and without ( $n=40$ trials, 2 rats, black) buspirone at (B) 1 week, (C) 2 weeks ( $n=20$ trials), (D) 6 weeks $(n=20$ trials), and (E) 8 wpi $(n=$ 20 trials). The reaching style and EMG activity patterns of these four rats reflected a standard, normal reaching strategy prior to injury; other EMGimplanted rats retrieved pellets with alternative dragging or looping strategies. Reach period was characterized by behavior as lift to arpeggio phases. Grasp period likewise was defined as grasp to release phases. Reach and grasp periods were normalized according to behavior characterization per attempt. There was reduced variance in the reach period among buspirone-treated rats from 2 to $6 \mathrm{wpi}$ in the pronator $\left(F_{(1,31)}=\right.$

paws closer to target pellets than that at pre-injury (Fig. 5). In contrast, buspirone-treated rats had elevated motor activity in the deltoid, extensor, and flexor during the single pellet task at 6 wpi. The motor activities of the extensor and flexor muscles were generally time locked to the reach and grasp periods of the task, respectively, in a similar manner to that of pre-injury. Unlike that of pre-injury, however, the biceps remained at a constant level of activity throughout the task (Fig. 4D). Buspirone-treated rats at 6 wpi consistently sustained low levels of continuous elbow flexion throughout the task and presented quick flexion of the wrist during the reach period of the task. The paw placement and reaching strategies of buspirone-treated rats at 6 wpi were accordingly more lateral and parabolic in relation to the target pellets than that at pre-injury (Fig. 5, Movie 7). Thus, daily serotonergic modulation facilitated reorganization of the neural networks, changing the relative levels of activation of motor pools and forming novel recruitment and coordination strategies to successfully reach and grasp pellets in the buspirone-treated rats.
3.116, $p=0.0874$, Levene's test $)$, extensor $\left(F_{(1,31)}=5.243, p=0.0290\right.$, Levene's test), and flexor $\left(F_{(1,31)}=5.719, p=0.0230\right.$, Levene's test $)$. Similarly, there was reduced variance in the grasp period among buspirone-treated rats from 2 to 6 wpi in the biceps $\left(F_{(1,31)}=4.623, p=\right.$ 0.0395 , Levene's test), extensor $\left(F_{(1,31)}=4.042, p=0.0532\right.$, Levene's test), and flexor $\left(F_{(1,31)}=6.638, p=0.0150\right.$, Levene's test). There was increased variance in the reach period among buspirone-treated rats from 6 to 8 wpi in the biceps $\left(F_{(1,56)}=4.228, p=0.0444\right.$, Levene's test $)$, extensor $\left(F_{(1,56)}=5.645, p=0.0210\right.$, Levene's test $)$, and flexor $\left(F_{(1,56)}=\right.$ $6.817, p=0.0116$, Levene's test). Similarly, there was increased variance in the grasp period among buspirone-treated rats from 6 to 8 wpi in the pronator $\left(F_{(1,56)}=5.831, p=0.0190\right.$, Levene's test $)$, extensor $\left(F_{(1,56)}=\right.$ $7.332, p=0.0090$, Levene's test $)$, and flexor $\left(F_{(1,56)}=8.412, p=\right.$ 0.0053 , Levene's test). All shaded regions indicate SEM.

Although the reaching and grasping scores of buspirone-tosaline-treated rats dropped from 6 to 8 wpi, the EMG patterns of these rats did not grossly change despite the switched treatment (Movie 8, Fig. 4E). Transitioning from buspirone treatment to saline treatment elicited slightly higher integrated EMG (iEMG) bursts from 6 to 8 wpi during the reach period and for the flexor during the grasp period. The timing of EMG bursts was generally not altered from the 6- to 8-week periods of the single pellet reaching task. Instead, the accuracy of reaching attempts had changed, and paw placement shifted closer to the rat or hovered above the pellet's location after transition from buspirone treatment to saline treatment (Movie 8). The saline-to-buspirone-treated rats also did not significantly alter EMG patterns during this transition (Fig. 4E). The iEMG amplitudes and periods were unchanged as was their performance rate.

To quantitatively assess the modulation in forelimb muscle recruitment over treatment and time, we compared the iEMG of muscle activities measured as the area under the 


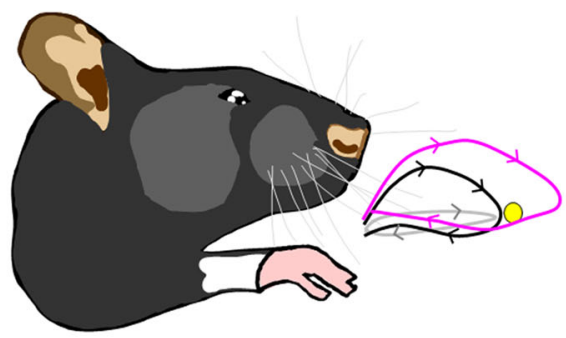

Saline Buspirone

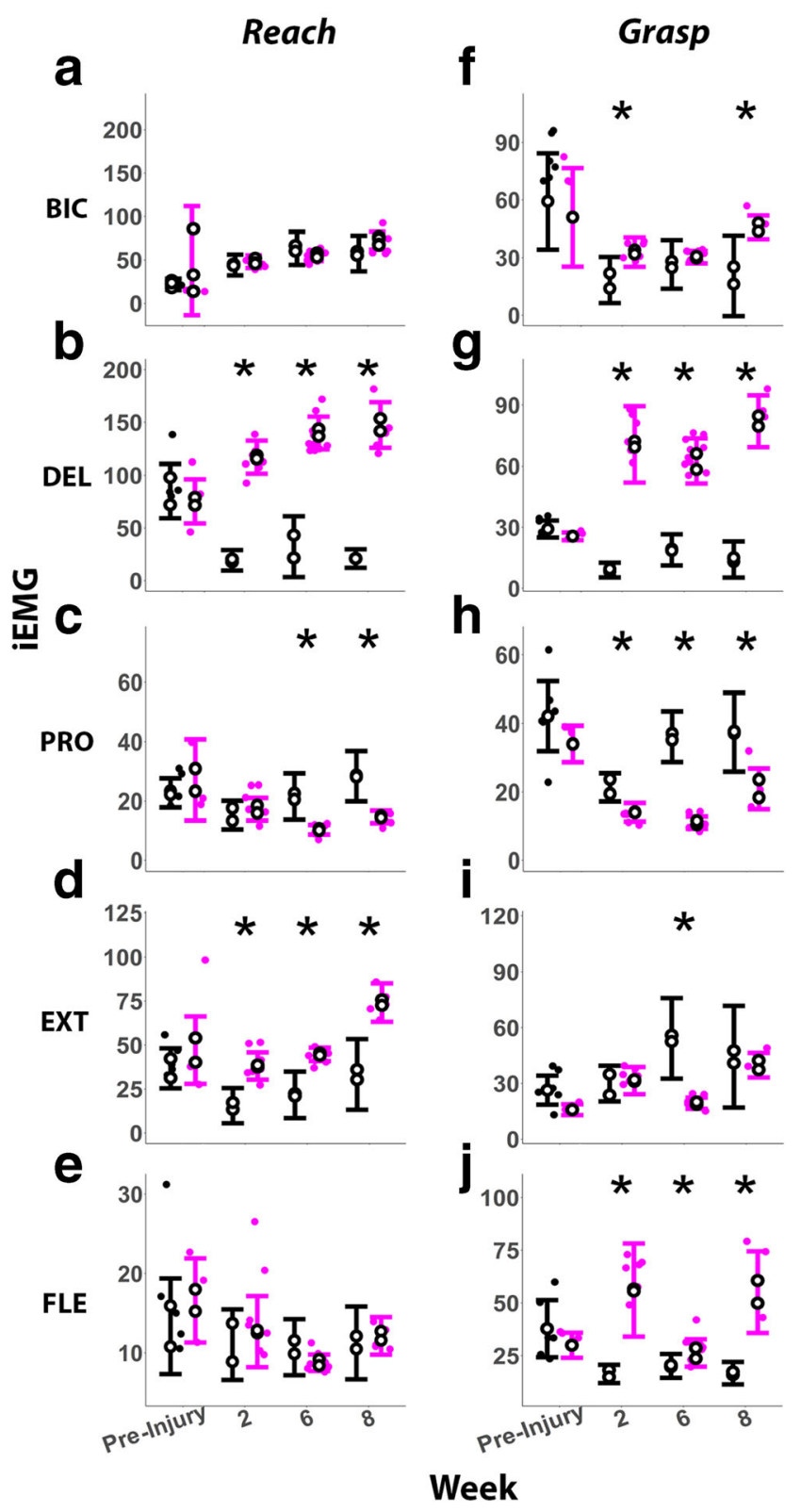

curve (AUC) during the reach (Fig. 5A-E) and grasp (Fig. 5F-J) periods of the single pellet reaching task. Cervical SCI decreased iEMG across all forelimb muscles during the task ( 4 of 5 muscles during the reach period and 4 of 5 muscles during the grasp period). Moreover, significant decreases in biceps and flexor muscle iEMGs were
Fig. 5 Integrated EMG (iEMG) activity from the dominant forelimb of four trained rats measured as the area under the curve (AUC) during (AE) reach (i.e., from lift to arpeggio stages of the task) and (F-J) grasp (i.e., from grasp to release stages of the task) periods of the single pellet reaching task before injury ( $n=20$ trials/animal) and with ( 2 rats, magenta) and without ( 2 rats, gray) buspirone at 2 wpi ( $n=20$ trials), 6 wpi $(n=$ 20 trials), and $8 \mathrm{wpi}$ ( $n=20$ trials). Prior to injury, these rats demonstrated a normal supination-pronation strategy (black trace in schematic). Following injury, rats generally demonstrated either a looping strategy consisting of overreaching the pellet (yellow) following buspirone administration (magenta trace) or a flattened trajectory following saline administration (gray trace). If an animal made multiple incomplete attempts consecutively, only the first attempt was scored as a trial and the sequential attempts were not considered for analysis. Significant changes were found in the average $\mathrm{iEMG}$ of the following muscles during the reach period: (A) deltoid (week 2: $F_{(2,148)}=11.962, p<0.0001$; week 6 : $F_{(2,148)}=14.826, p<0.0001$; week 8: $F_{(2,148)}=22.706, p<0.0001$; ANOVA), (B) pronator (week 6: $F_{(2,148)}=4.834, p<0.0001$; week 8: $F_{(2,148)}=7.703, p<0.0001 ;$ ANOVA), and (C) extensor digitorum (week $2: F_{(2,148)}=4.297, p<0.0001$; week $6: F_{(2,148)}=4.956, p<0.0001$; week 8: $F_{(2,148)}=11.435, p<0.0001$; ANOVA). Significant changes found in the average iEMG of the following muscles during the grasp period: $(\mathrm{F})$ deltoid (week 2: $F_{(2,136)}=13.332, p<0.0001$; week 6: $F_{(2,136)}=10.613$, $p<0.0001$; week 8: $F_{(2,136)}=21.496, p<0.0001$; ANOVA), (G) biceps (week 2: $F_{(2,136)}=2.668, \mathrm{p}=0.009$; week 8: $F_{(2,136)}=6.778, p<0.0001$; ANOVA), $(\mathrm{H})$ flexor digitorum (week $2: F_{(2,136)}=6.701, p<0.0001$; week 8: $F_{(2,136)}=9.492, p<0.0001$; ANOVA), (I) Extensor digitorum (week 6: $F_{(2,136)}=6.959, p<0.0001$; ANOVA), and (J) pronator (week 2: $F_{(2,136)}=2.614, p=0.010$; week $6: F_{(2,136)}=10.541, p<0.0001$; week 8: $F_{(2,136)}=8.672, p<0.0001 ;$ ANOVA). There was increased variance in iEMG among all forelimb motor pools excluding that of the deltoid in buspirone-to-saline SCI animals from 6 to 8 wpi: biceps $\left(F_{(1,172)}=\right.$ 5.4513, $p=0.021$; Levene's test $)$, pronator $\left(F_{(1,172)}=11.268, p=\right.$ 0.0009; Levene's test), flexor $\left(F_{(1,172)}=32.334, p<0.0001\right.$; Levene's test), and extensor $\left(F_{(1,172)}=15.367, p=0.0001\right.$; Levene's test $)$. Animals were divided into saline (gray) and buspirone (magenta) and received their respective treatment at 1-6 wpi. iEMG values for successful reaches are noted as smaller, filled dots per time point. Average iEMG values per animal are noted as larger white dots.

observed in saline- but not buspirone-treated animals after injury (Fig. 5F, J). Acute buspirone was sufficient to reverse the bulk of this effect by 2 wpi (Fig. 5B, D, F, G, J). Nevertheless, buspirone did not increase pronator iEMG and instead further depressed pronator iEMG values during the task over multiple weeks (Fig. 5C, H).

Following SCI, all forelimb muscles in buspirone-treated rats demonstrated less variance in iEMG values for successful reaches than for failed reaches, displaying consistent use of certain strategies for success ( 3 of 5 muscles showed significance: biceps, $F_{(1,68)}=7.130, p<0.0001$; deltoid, $F_{(1,68)}=$ $2.853, p=0.0046$; pronator, $F_{(1,68)}=2.524, p=0.0101$; Levene's test). If there was a buspirone-induced change in reaching strategy, the novel functional networks would most likely readapt and then adapt across drug treatment (i.e., 2 to 6 wpi), showing increased and reduced variance in EMG values across individual motor pools, respectively. The variance of iEMG values from saline-treated rats at 2 to $6 \mathrm{wpi}$ and during the saline-to-buspirone treatment switch at 6 to 8 wpi remained mostly consistent with exception to that of the deltoid muscle, 
denoting that a similar reaching strategy was used by salinetreated rats before and after injury. In general, the motor pools that are primarily activated during the reach and/or grasp periods prior to injury were selectively enhanced after buspirone.

\section{Pharmacological Modulation of Spinal Neural Connectivity Reflected in sMEPs}

sMEPs were collected from rats at rest by applying lowfrequency epidural electrical stimulation at $2 \mathrm{~Hz}$ to spinal levels C6 and C8 as demonstrated previously $[15,17]$ to characterize spinal cord-to-motor pool circuitry after injury and buspirone treatment. We monitored sMEPs only after all behavioral testing for each week was complete and for $30 \mathrm{~min}$ per week to prevent a stimulation effect on reaching ability. sMEPs were divided into early ( $<10 \mathrm{~ms}$ latency) and late $(10$ $30 \mathrm{~ms}$ latency) responses. sMEPs were evoked in awake, resting rats with a constant current protocol in the $100-1000 \mu \mathrm{A}$ range. sMEP threshold per animal, or the current at which an animal first elicited a behavioral response in any of the selected forelimb muscles, did not change significantly over time, injury, or treatment. However, the SMEP threshold value and number of muscles responding to sMEP threshold were different between animals (Fig. 6A, D). These differences could
Fig. 6 Representative normalized spinal motor-evoked responses (sMEPs) from a single rat of selected forelimb muscles from the saline-treated and buspironetreated groups to $2 \mathrm{~Hz}$ (yellow arrow) spinal cord epidural stimulation when the rats were standing still (60-80 pulses/animal/ time point, $\mathrm{C} 6+\mathrm{C} 8$ bipolar stimulation, constant current at $400 \mu \mathrm{A})$. The area under the curve (AUC) of sMEPs were quantified in 0.05 time bin and plotted per muscle channel below their respective sMEP plots. These data were normalized per animal to their pre-injury mean responses and then averaged per each time point: (A, D) pre-SCI, $(\mathrm{B}, \mathrm{E}) 1 \mathrm{wpi}$, and $(\mathrm{C}, \mathrm{F}) 6$ wpi. At $1 \mathrm{wpi}$, animals were divided into two groups, receiving saline ( $n=$ $5)$ or buspirone $(n=5)$ treatment for 1-6 wpi. sMEP responses were divided into early responses (ER, 0-10 ms after pulse) and late responses (LR, 10-30 ms after pulse) for analysis. All shaded regions indicate SEM.

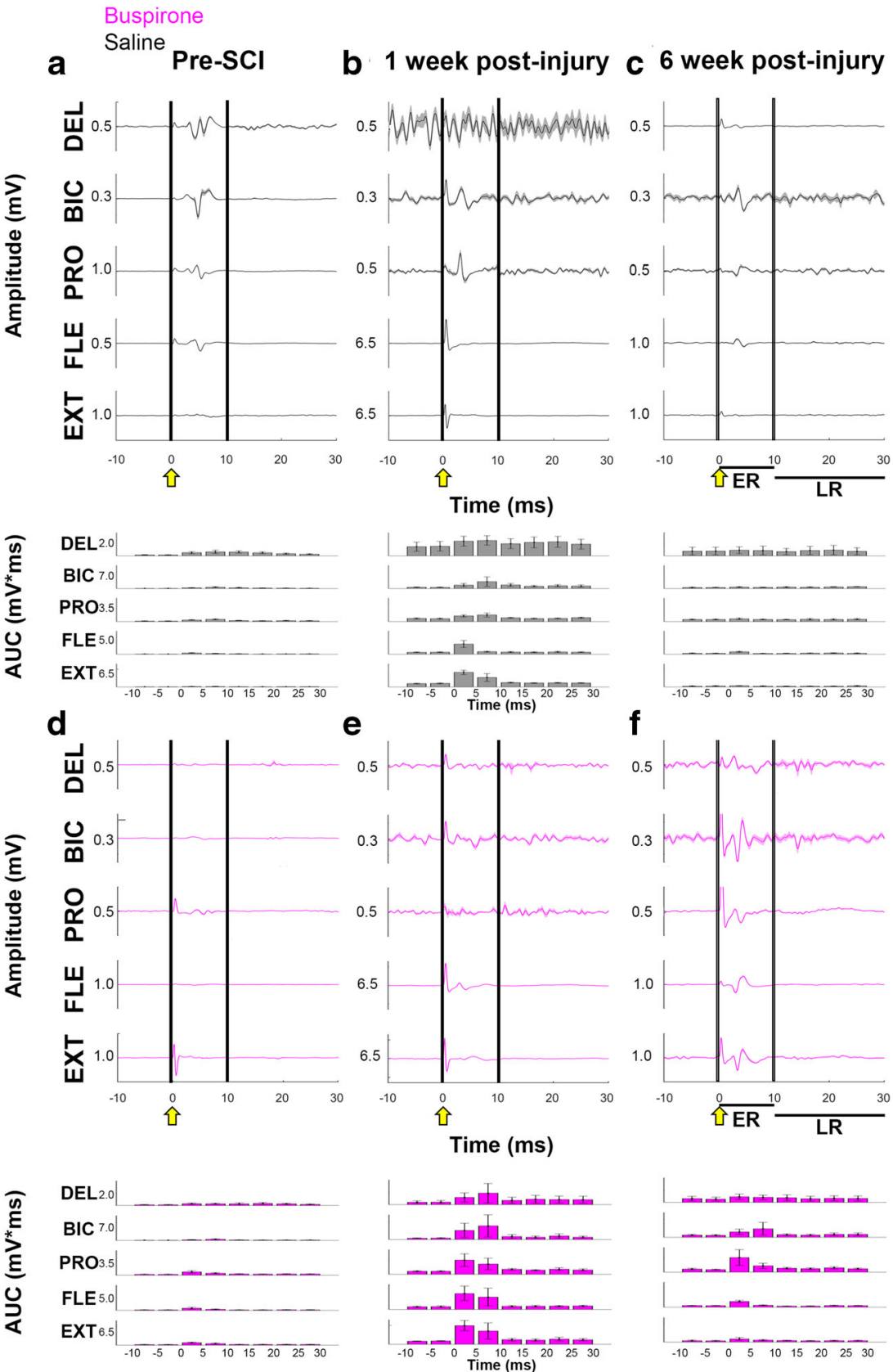


be contributed to individual differences in behavior response and stimulation. Accordingly, sMEP responses were normalized to pre-injury values, and the amount of change in SMEPs was evaluated to highlight electrophysiological changes in forelimb motor pools across time and treatment. We computed 3 parameters of sMEP responses using a custom written MATLAB script: motor latency, AUC, and peak-to-peak (P2P) amplitude. When comparing averaged sMEP responses for each individual set of stimulation configuration and current parameters (e.g., sMEP threshold), cervical SCI increased the amplitude and AUC values of motor pool responses for all muscles (Fig. 6A, B). The basal activity of the deltoid muscle especially increased in response to sMEP stimulation. Acute buspirone administration at 1 wpi slightly increased the amplitude and AUC values of sMEP responses at the same stimulation parameters (Fig. 6E, 4 of 5 muscles). At 6 wpi, the amplitude and AUC of sMEPs to the same stimulation parameters were significantly reduced in saline-treated animals but more sustained in buspirone-treated animals (Fig. 6C, F). The level of basal activity in the extensor motor pools was elevated in all animals at 6 wpi compared to that of pre-injury, particularly among the buspirone-treated animals (Fig. 6C, F, bar charts). Although sMEPs for each individual set of stimulation parameters were generally increased after acute SCI, the elevated sMEP response to the same set of stimulation parameters was only maintained in buspirone-treated rats by 6 wpi.

To distinguish overall differences in early and late sMEP responses, sMEPs for all stimulation configurations and currents - including those below and above threshold - were normalized and averaged to pre-injury values per animal across time and treatment. There were no significant differences in the latency of any sMEP responses across muscles at any time point, including between pre- and post-injury. Buspirone administration following cervical SCI decreased early sMEPs of extensor and pronator motor pools compared to that of saline administration (Fig. 7A, B). In contrast, buspirone increased AUC in late sMEPs of biceps, extensor, and flexor motor pools compared to that of saline treatment (Fig. 7C-E). Buspirone modulated muscle excitability in the forelimb following SCI.

\section{Serotonergic Treatment in SCI Rats with Forelimb Reaching Training Does Not Improve Locomotor Function}

To determine whether improvements in reaching and grasping translated into improvements on motor tasks requiring motor control of the hindlimbs as well as forelimbs, SCI rats were subjected weekly to a horizontal ladder walking task with unevenly spaced rungs [33]. Forelimb footsteps were scored and summed (adapted from Metz and Whishaw [33]) as successful placements (correct, partial) or as erroneous placements (slip, miss). The number of foot faults over three consecutive sessions per time point was averaged and compared between buspironeand saline-treated rats. In contrast to the single pellet task, incomplete SCI rats rarely produced errors in their forelimbs during locomotion (Fig. 8). Buspirone-treated rats showed an increase in successful partial foot placements at 3 wpi $\left(F_{(3,74)}=\right.$ 2.184, $p=0.032$, ANOVA), but this difference was lost between treatments by 6 wpi. Improvement in fine forelimb reaching and grasping with buspirone or fluoxetine did not transfer into improvements in locomotion.

\section{Discussion}

Both serotonergic agents promoted forelimb recovery after cervical SCI. Partial $5 \mathrm{HT}_{1 \mathrm{~A}} \mathrm{R}$ agonist buspirone and SSRI fluoxetine facilitated reaching and grasping. Buspirone plateaued performance within 2 weeks while fluoxetinetreated rats continued to improve over an 8-week period. Second, buspirone-treated rats showed robust changes in muscle recruitment patterns for success during skilled tasks compared to saline-treated rats. The muscle (motor pool) recruitment patterns were distinctly different in multiple features between pre- and post-injury with and without pharmacological intervention. Third, serotoninergic modulation enabled rapid and progressively refined patterns of functional reorganization of neural networks, providing effective regulatory control of what must be considered to be de novo or at least novel supraspinal-spinal, functional connectomes. Fourth, the advancements made in forelimb skilled tasks did not transfer to a locomotor-ladder task.

\section{Serotoninergic Agents Facilitate Spinal Networks that Execute Reaching and Grasping}

Serotonergic intervention modulates forelimb motor pools to facilitate functional recovery in a time-sensitive and drugspecific manner. Previous studies have shown buspirone facilitated locomotion in spinal cord-transected mice with training alone [34], and also with carbidopa and L-Dopa [21]. Fluoxetine can modulate spinal cord excitatory/inhibitory neurotransmitter levels in SCI animals via a variety of mechanisms including neurogenesis, gliogenesis, synaptogenesis, and cortical mapping by blocking 5HT reuptake and stimulating residual somatodendritic connections [6, 35-39]. Enhancement of 5HT response among surviving descending brainstem 5HT axons could further facilitate forelimb recovery [40]. Conversely, buspirone is unlike fluoxetine in that $5 \mathrm{HT}_{1 \mathrm{~A}} \mathrm{R}$ activation can excite or inhibit neuromotor networks depending on the target or location, potentiating cellular excitability while suppressing plateaued activity [34]. This difference could underlie the plateaued and gradual forelimb performance with buspirone and fluoxetine, respectively, after SCI. The present data combined with previous studies of 
Fig. 7 Early and late normalized sMEPs of selected forelimb muscles to spinal cord epidural stimulation when the rats were resting quietly. sMEP parameters were normalized per animal to their pre-injury responses and then plotted on a log scale. Significant differences between the two treatment groups were found in late sMEPs for the parameter $\mathrm{IEMG}$ in the (A) biceps (week 1: $F_{(2,298)}=2.333, p=$ 0.020 ; week 6: $F_{(2,298)}=2.820$, $p=0.005$; ANOVA), (B) flexor (week 1: $F_{(2,298)}=3.637, p=$ 0.0003; week 6: $F_{(2,298)}=2.061$, $p=0.040$; ANOVA), and (C) extensor $\left(\right.$ week 1: $F_{(2,298)}=3.814$, $p=0.0002$, ANOVA) muscles. All error bars indicate SEM. Similar differences were found for the parameter peak-to-peak amplitude of sMEPs (P2P; refer to "Materials and Methods") in the same muscles. Individual iEMG values are noted as small, filled dots. Average iEMG values per animal are noted as larger white dots. Other selected forelimb muscles did not display significant results for late sMEP parameters.

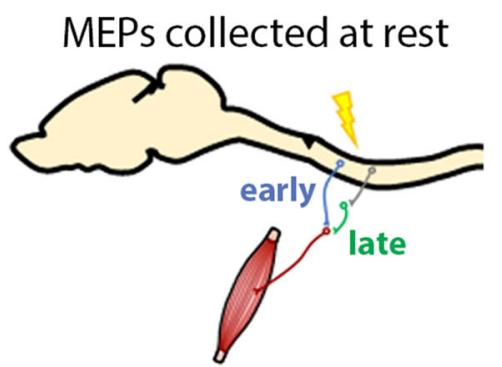

\section{Saline Buspirone}
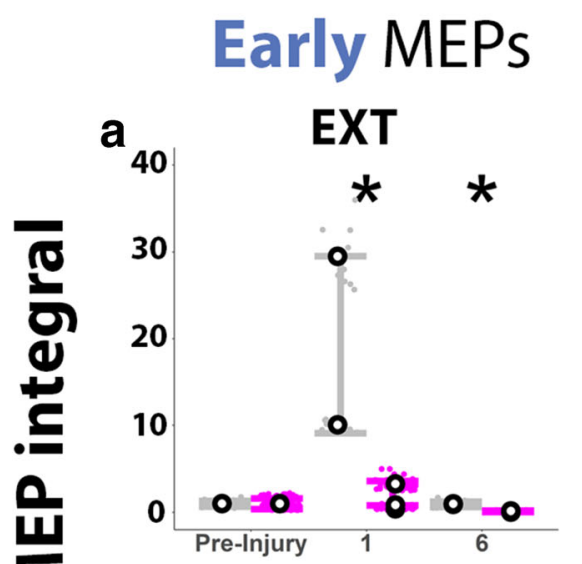

ํㅗㄹ
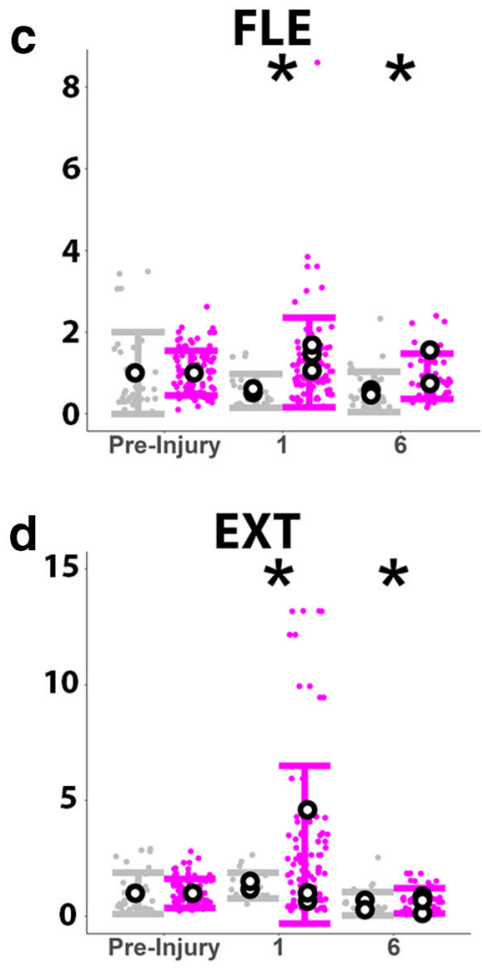

e

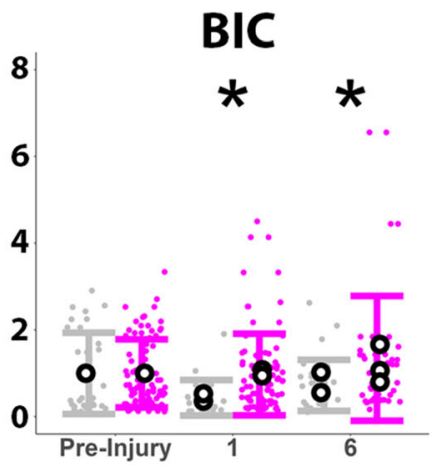

motor systems provides a compelling case for careful and systematic testing the efficacy of serotonergic agents to facilitate recovery of motor function following cervical spinal injury in humans.

The present study was designed to begin to elucidate some of the dose-related effects of serotonergic drugs on motor recovery after SCI. Fluoxetine differentially affected forelimb performance based on whether the dosage started at a higher fixed dose or lower, but gradually increasing dose following spinal cord injury. Even though the final dose in both experiments was the same at $5 \mathrm{mg} / \mathrm{kg} /$ day by 6 wpi, the increasing dose paradigm with fluoxetine was not sufficient to rescue reaching and grasping ability. One potential explanation for this marked difference in forelimb performance is the need for a longer acclimation time for fluoxetine in the spinal cord to modulate neural networks and evoke an enhanced behavioral response in the forelimb. Fluoxetine has a protracted effect on locomotor performance even at a higher dose of $10 \mathrm{mg} / \mathrm{kg}$ following SCI [41]. Forelimb performance continued to improve at a fixed $5 \mathrm{mg} / \mathrm{kg} /$ day dose until 8 wpi, achieving a forelimb performance similar to or even better than at preinjury, suggesting that the effect of fluoxetine on the supraspinal-spinal networks for the forelimb could be attributed to 1) a more gradual and persistent effect than that of buspirone, which instead evoked an exponential increase in reaching and grasping scores by 2 wpi and a plateaued performance until $6 \mathrm{wpi}$, and/or 2) recruiting different neural networks for reaching and grasping. 


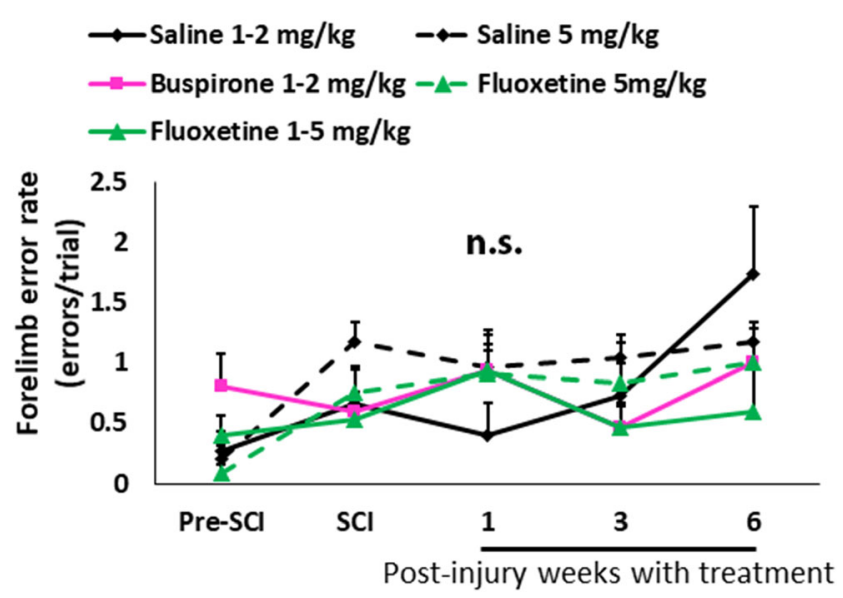

Fig. 8 Buspirone and fluoxetine administration did not reduce error rate in SCI rats walking across a horizontal ladder. No significant differences were found between buspirone-treated ( $n=5$, magenta), incremental fluoxetine-treated ( $n=5$, green, solid), fixed fluoxetine-treated $(n=7$, green, dashed), incremental saline-treated ( $n=5$, black, solid), and fixed saline-treated ( $n=7$, black, solid) rats pre- and post-injury.

Considering their primarily clinical roles as antidepressants, both buspirone and fluoxetine are likely to act on circuits underlying motivation. They can differentially modulate motivated behaviors depending on its dose and context [42-47]. Motivation can highly affect motor performance. Previous work by the Courtine lab has shown that using chocolate as a food incentive encouraged SCI rats to locomote in addendum to an electrochemical neuroprosthesis [5]. However, we should note that all rats in this study were food deprived and given the same sugar pellets as reward in the forelimb reaching task. All rats were scored by raters blind to drug treatment. We assume that the level of motivation realized by each rat was randomly distributed. Moreover, no overt behavioral differences were observed that reflected a significant difference in the drive to retrieve the food pellets nor their skill as measured as a percent of the efforts that were successful. These data suggest that coordinating the recruitment of spinal and supraspinal networks, at least in part, underlies the improved forelimb performance after SCI, but do not exclude a role for motivation.

Based on the differences in the pattern of improvement in response to the two agents tested, further investigation on the mechanisms of these two serotonergic agents on supraspinalspinal networks at different stages of SCI could provide more predictable and effective dosage strategies for facilitating skilled movements in the arm after injury. Buspirone has been shown to enhance the benefits of stimulation and training in handgrip function and locomotor movement in chronic SCI human subjects $[8,48]$, but has yet to be tested at multiple doses in a longitudinal study. Less is known about the effect of fluoxetine in facilitating movement in paralyzed patients. Fluoxetine enhanced the benefit of repetitive transcranial magnetic stimulation in stroke patients for forelimb function [49], but it is unknown about its electrophysiological effects on spinal circuits after neural trauma in humans. In summary, the present data on dose effectiveness suggest that both the absolute dosage, e.g., as with buspirone, and the pattern and duration of the dosage, e.g., fluoxetine, are important to consider in its use as an intervention.

\section{EMG Patterns Serve as Physiological Biomarkers of Motor Pool Specific Network Reorganization}

We propose that an important phenomenon emerged in the changes found in forelimb performance and EMG patterns. The networks essential for forelimb reaching and grasping could have re-entered a critical period of permissiveness to mechanisms of plasticity, similar to that of which occurs during development. Despite showing a performance plateau within 2 weeks, buspirone-treated rats continued to exhibit dynamic changes in the timing and amplitude of EMG responses that emerged during the 6 weeks of treatment. These changes were gradually reflected in reaching and grasping behavior, generating different reaching strategies and trajectories over time and treatment. Furthermore, the changes in interneuronal connectivity specific to forelimb motor pools occurred for at least $6 \mathrm{wpi}$, suggesting a continuous remodeling of neuronal and synaptic groups of networks, a phenomenon consistent with Edelman's activity-dependent neural Darwinism hypothesis [50]. A component of Edelman's hypothesis theorizes that neural circuits continuously 1) update spatial and temporal representation of sensory information, and 2) this update is brought about by a continuing selection of neuronal ensembles that translates the continuous changing of sensory inputs into a predictable, but not determinate motor synergies. Theoretically, after a spinal injury, the neuronal ensembles will consist of a number of functionally aberrant synaptic connections and poor coordination. However, when these ensembles are repeatedly activated, they can selforganize in a use-dependent manner by functionally pruning the aberrant synapses, thus resulting into a more skilled and less variable reaching and grasping behavior. This concept therefore provides a mechanism through which different neuronal groups can accomplish the same function, a phenomenon often referred to as redundancy networks [50, 51]. Considering the changes in EMG patterns during reaching and grasping and increased spinal excitability at $6 \mathrm{wpi}$, buspirone after SCI may facilitate remodeling of spinal networks important for forelimb function. The data demonstrate a recovery process consisting of multiple and probably partially overlapping adaptive strategies, with each adaptive mechanism occurring over different time frames.

A second observation is that the neural network adaptations that occur in response to injury and treatment can substantially vary by individual and time point; i.e., multiple reaching strategies can accomplish the same task. The variety of reaching strategies found in this study strengthens the idea that there are 
multiple ways to recruit forelimb motor pools to accomplish the same skilled task. This seems to have been particularly the case when trying to complete tasks after a severe loss of the original neural components, in this case the dCST for reaching and grasping. The success rate of buspirone-treated rats in the single pellet reaching task after SCI appears to be linked with the changes in their EMG patterns and reaching strategies. For the same reason, however, a therapeutic intervention could differentially interact with neuromotor circuits based on the state of the individual's reaching and grasping strategy specific to that time point. The same treatment could induce different effects on behavior based on the timing of its application. Our data demonstrate a critical window of plasticity permissive to buspirone facilitation after acute spinal cord injury. This may be partially lost in more chronic SCI patients, who require pairing with additional treatment to see forelimb improvement [48]. The results suggest that recovering normal biomechanics, i.e., similar to pre-injury, is a lower priority than the ability to effectively regain a function, regardless of different biomechanical strategies.

Thirdly, motor variability is reduced and success rate is improved during reaching and grasping following buspirone treatment. Variance in forelimb iEMG values decreased from 2 to 6 wpi following daily buspirone administration and increased following withdrawal at 6-8 wpi in buspirone-to-saline-treated rats, which correlated to changes in success scores of performance. We similarly show that successful execution of the single pellet task before and after injury was associated with lower motor variability than that of failed attempts (Movies 9 and 10). These data suggest that discovering a successful strategy to execute specific motor patterns results in gradually forming more stable "synergisms" among sensory-motor neural networks that increase the probability of success [52].

Our results resemble studies in which experts of complex tasks such as triple-jumping and beam-walking show lower variability in motor errors and higher consistency of motor outcome than novices [53, 54]. Highly trained animals are unlikely to deviate from an operational and previously used strategy unless they undergo extinction or reversal training [55]. The degree of variance in motor output is an important factor in predicting reaching and grasping success. Interestingly, a caveat to this principle emerges after introducing buspirone at 1 wpi. Recordings of reaching behavior in buspirone-treated rats display a distinct shift in reaching trajectory and strategy matched by dynamic changes in EMG patterns and increase in variance of iEMG values (Fig. 4). In line with the Edelman hypothesis, serotonergic intervention in combination with injury could have induced a state in the spinal cord mimicking the critical period that occurs during neonatal development, in which a high degree of movementto-movement variability allows exploration of different strategies and facilitates adaptive learning to a changing environmental state [56].

\section{Potential Mechanisms of Monoaminergic Modulation of Cervical Networks}

Buspirone is a primarily anxiolytic drug with high affinity for $5 \mathrm{HT}_{1 \mathrm{~A}} \mathrm{Rs}$, weak affinity for $5 \mathrm{HT}_{2} \mathrm{Rs}$, and antagonistic properties against dopamine D2Rs; diversifying the several mechanisms in which buspirone can modify monoaminergic neurotransmission $[57,58]$. Low dosages of buspirone ( $1 \mathrm{mg} / \mathrm{kg}$ ) can decrease $5 \mathrm{HT}$ turnover without significantly decreasing motor activity, stimulating somatodendritic $5 \mathrm{HT}_{1 \mathrm{~A}} \mathrm{Rs}$ that produce inhibitory regulation on dopaminergic neurotransmission and modulate motoneurons in the spinal cord [59]. Moreover, $5 \mathrm{HT}_{1 \mathrm{~A}} \mathrm{R}$ agonist application after SCI can upregulate $\mathrm{KCC} 2$ co-transporters and reverse the transformation of GABA-ARs from inhibitory to excitatory neural effectors and effectively reverse SCI effects [60]. Altering inhibitory regulation in the intact spinal cord perturbs forelimb reaching, indicating its importance in coordinating smooth execution of skilled movements [61]. Interestingly, there are generally more forelimb oscillations during the reaching and grasping attempts, e.g., during extension and retrieval of pellets in the present study at $8 \mathrm{wpi}$ compared to that of $6 \mathrm{wpi}$, following a switch in buspironeto-saline treatment. Although more effort is necessary to fully understand the effect of buspirone on neuromotor networks, our observation that buspirone differentially recruits motor pools as well as modulate evoked responses in forelimb behavior suggests that, at least in spinal circuitry for forelimb, buspirone induces functional plasticity via alteration of interneuronal connectivity - potentially affecting excitation/inhibition balance-important for reaching and grasping.

We propose that the changes in sMEPs after buspirone reflect significant functional reorganization and refinement of the brain-to-spinal networks. Although acute SCI increased the amplitude and number of sMEPs, buspirone administration over a 6-week period evoked more forelimb muscle excitability than that of saline in sub-acute SCI. Aberrant functional connectivity and spasticity at $1 \mathrm{wpi}$ could result in the loss of function by 6 wpi if not treated soon after injury. Daily buspirone administration further potentiated the likelihood of sMEP responses by increasing the basal level of spinal network activity for the selected forelimb motor pools at the acute and subacute injury phases. As ERs are likely dominated by monosynaptic activation of motoneurons while LRs are derivative of secondary polysynaptic communication predominantly through spinal interneuron networks, an increase and decrease in the LRs and ERs following buspirone suggest serotonin as a key neuromodulator of residual interneuronal circuitry after injury [62]. Considering that buspirone also enhanced sMEP excitability and induced EMG patterns distinct from pre-injury rats, it is unlikely that buspirone only enhanced 
local serotonin levels and not that of spinal networks underlying forelimb function. These data point to the observation that the final key factor in recovering successful reaching and grasping is the ability to reacquire a strategy for reorganizing the spinal interneuronal networks so that the motor pools can effectively coordinate the key motor pools. It is not a matter so much as to whether the motor pools can be recruited, but the temporal patterns of activation of those motor pools as determined by the interneurons that functionally project to those motor pools.

Although the present data do not demonstrate the degree of a specific activity pattern necessary to facilitate improved task performance, the results consistently show an element of facilitation and marked changes in patterns of coordination of forelimb networks during reaching and grasping behavior. This is of importance given that the rats were tested weekly, but not trained to reach and grasp after injury. The rats, however, obviously used similar movements during routine daily feeding behavior. For this reason, we cannot determine from the present data the degree to which the recovery of function can be attributed to the amount or specificity of activity-dependent mechanisms shaping the behavior tested during the post lesion period. We have evidence, however, that in an analogous scenario when paralyzed rats are stimulated with epidural stimulation below motor threshold for $5 \mathrm{~h}$ continuously while in their normal cage environment, they are five times more active than when there was no stimulation [63]. We suspect that the results were similar in the present study when there was a chronic neuromodulation occurring pharmacologically. This interpretation is consistent with the many reports demonstrating a critical role for activity dependence being a key factor in the functional reorganization, but not knowing the degree of specificity necessary in the neuromodulation of practicing a motor task [64-66]. It is also consistent with Edelman's theory of the theory of neuronal group selection followed by use-dependent plasticity as a means of guiding synaptic reorganization [50]. Thus, we propose that 5HTR activation potentiates synaptic plasticity and practice via spontaneous activity guided the synergistic reorganization within and among supraspinal and spinal networks to regain more effective and coordinated functionality.

Supplementary Information The online version contains supplementary material available at https://doi.org/10.1007/s13311-020-00974-8.

Acknowledgments This research was supported by the National Institute of Biomedical Imagining and Bioengineering (NINDS 1U01EB015521), Broccoli Foundation, the Christopher \& Dana Reeve Foundation, and the RFBR No. 13-04-12030, and by Russian Scientific Fund project No. 1445-00024.

Required Author Forms Disclosure forms provided by the authors are available with the online version of this article.

\section{References}

1. Kiehn O. Locomotor circuits in the mammalian spinal cord. Annu Rev Neurosci 2006;29:279-306.

2. Zhang M. Normal Distribution and Plasticity of Serotonin Receptors after Spinal Cord Injury and Their Impacts on Motor Outputs. In: Recovery of Motor Function Following Spinal Cord Injury. InTech; 2016.

3. Han Q, Cao C, Ding Y, So K-F, Wu W, Qu Y, et al. Plasticity of motor network and function in the absence of corticospinal projection. Exp Neurol. 2015 May 1 [cited 2019 Jun 9];267:194-208. Available from: https://www.sciencedirect.com/science/article/pii/ S001448861500076X?via\%3Dihub

4. Cabaj AM, Majczyński H, Couto E, Gardiner PF, Stecina K, Sławińska U, et al. Serotonin controls initiation of locomotion and afferent modulation of coordination via 5-HT7 receptors in adult rats. J Physiol. 2017 Jan 1 [cited 2019 Nov 3];595:301-20. Available from: http://doi.wiley.com/10.1113/JP272271

5. Van Den Brand R, Heutschi J, Barraud Q, DiGiovanna J, Bartholdi $\mathrm{K}$, Huerlimann M, et al. Restoring voluntary control of locomotion after paralyzing spinal cord injury. Science (80- ) 2012;336:11825.

6. Scali M, Begenisic T, Mainardi M, Milanese M, Bonifacino T, Bonanno G, et al. Fluoxetine treatment promotes functional recovery in a rat model of cervical spinal cord injury. Sci Rep 2013;3.

7. Ung R V., Landry ES, Rouleau P, Lapointe NP, Rouillard C, Guertin PA. Role of spinal 5-HT2 receptor subtypes in quipazineinduced hindlimb movements after a low-thoracic spinal cord transection. Eur J Neurosci 2008;28:2231-42.

8. Sławínska U, Miazga K, Jordan LM. 5- $\mathrm{HT}_{2}$ and 5-HT 7 receptor agonists facilitate plantar stepping in chronic spinal rats through actions on different populations of spinal neurons. Front Neural Circuits 2014;8.

9. Gad P, Gerasimenko Y, Zdunowski S, Turner A, Sayenko D, Lu DC, et al. Weight bearing over-ground stepping in an exoskeleton with non-invasive spinal cord neuromodulation after motor complete paraplegia. Front Neurosci 2017;11.

10. K. Scullion, J. A. Boychuk, G. R. Yamakawa, J. T. G. Rodych, S. T. Nakanishi, A. Seto, V. M. Smith, R. W. McCarthy, P. J. Whelan, M. C. Antle, Q. J. Pittman, G. C. Teskey, (2013) Serotonin 1A Receptors Alter Expression of Movement Representations. Journal of Neuroscience 33 (11):4988-4999

11. Pavel D. Hrdina, Thu B. Vu, (1993) Chronic fluoxetine treatment upregulates 5-HT uptake sites and 5-HT2 receptors in rat brain: An autoradiographic study. Synapse 14 (4):324-331

12. Alfredo Meneses, Enrique Hong, (1995) Effect of fluoxetine on learning and memory involves multiple 5-HT systems. Pharmacology Biochemistry and Behavior 52 (2):341-346

13. Peng L, Gu L, Li B, Hertz L. Fluoxetine and all other SSRIs are 5$\mathrm{HT}_{2 \mathrm{~B}}$ Agonists - Importance for their Therapeutic Effects. Curr Neuropharmacol 2014;12:365-79.

14. Wang Y, Neumann M, Hansen K, Hong SM, Kim S, NobleHaeusslein LJ, et al. Fluoxetine increases hippocampal neurogenesis and induces epigenetic factors but does not improve functional recovery after traumatic brain injury. J Neurotrauma 2011:28:259-68.

15. Alam M, Garcia-Alias G, Jin B, Keyes J, Zhong H, Roy RR, et al. Electrical neuromodulation of the cervical spinal cord facilitates forelimb skilled function recovery in spinal cord injured rats. Exp Neurol 2017;291:141-50.

16. Roy RR, Hutchison DL, Pierotti DJ, Hodgson JA, Edgerton VR. EMG patterns of rat ankle extensors and flexors during treadmill locomotion and swimming. J Appl Physiol 1991;70:2522-9.

17. Alam M, Garcia-Alias G, Shah PK, Gerasimenko Y, Zhong H, Roy $\mathrm{RR}$, et al. Evaluation of optimal electrode configurations for 
epidural spinal cord stimulation in cervical spinal cord injured rats. J Neurosci Methods 2015;247:50-7.

18. Jin B, Alam M, Garcia-Alias G, et al. Buspirone treatment promotes forelimb functional recovery in cervical spinal cord injured rats. In: Society for Neuroscience Chicago Nanosymposium Advances in SCI Research and Plasticity. 2016 [cited 2020 May 21]. p. 197.08. Available from: https:/www.researchgate.net/publication/ 318760604 Buspirone treatment promotes forelimb functional recovery_in_cervical_spinal_cord_injured_rats

19. Cristante AF, Filho TEPB, Oliveira RP, Marcon RM, Ferreira R, Santos GB. Effects of antidepressant and treadmill gait training on recovery from spinal cord injury in rats. Spinal Cord 2013;51:5017.

20. Engesser-Cesar C, Anderson AJ, Cotman CW. Wheel running and fluoxetine antidepressant treatment have differential effects in the hippocampus and the spinal cord. Neuroscience. 2007;144:103344.

21. Ung RV, Rouleau P, Guertin PA. Functional and physiological effects of treadmill training induced by buspirone, carbidopa, and L-DOPA in clenbuterol-treated paraplegic mice. Neurorehabil Neural Repair 2012;26:385-94.

22. Guertin PA, Ung RV, Rouleau P, Steuer I. Effects on locomotion, muscle, bone, and blood induced by a combination therapy eliciting weight-bearing stepping in nonassisted spinal cord-transected mice. Neurorehabil Neural Repair 2011;25:234-42.

23. Whishaw IQ, Dringenberg HC, Comery TA. Rats (Rattus norvegicus) modulate eating speed and vigilance to optimize food consumption: effects of cover, circadian rhythm, food deprivation, and individual differences. J Comp Psychol 1992;106:411-9.

24. Piecharka DM, Kleim JA, Whishaw IQ. Limits on recovery in the corticospinal tract of the rat: Partial lesions impair skilled reaching and the topographic representation of the forelimb in motor cortex. Brain Res Bull. 2005 Aug 15 [cited 2019 Jun 9];66:203-11. Available from: https://www.sciencedirect.com/science/article/pii/ S0361923005001784?via\%3Dihub\#fig3

25. García-Alías G, Truong K, Shah PK, Roy RR, Edgerton VR. Plasticity of subcortical pathways promote recovery of skilled hand function in rats after corticospinal and rubrospinal tract injuries. Exp Neurol 2015;266:112-9.

26. McKenna JE, Whishaw IQ. Complete compensation in skilled reaching success with associated impairments in limb synergies, after dorsal column lesion in the rat. J Neurosci 1999;19:1885-94.

27. Girgis J, Merrett D, Kirkland S, Metz GAS, Verge V, Fouad K. Reaching training in rats with spinal cord injury promotes plasticity and task specific recovery. Brain. 2007 Apr 5 [cited 2019 Nov 3];130:2993-3003. Available from: https://academic.oup. com/brain/article-lookup/doi/10.1093/brain/awm245

28. Solnik S, Rider P, Steinweg K, Devita P, Hortobágyi T. TeagerKaiser energy operator signal conditioning improves EMG onset detection. Eur J Appl Physiol 2010;110:489-98.

29. Shah PK, Garcia-Alias G, Choe J, Gad P, Gerasimenko Y, Tillakaratne N, et al. Use of quadrupedal step training to reengage spinal interneuronal networks and improve locomotor function after spinal cord injury. Brain. 2013 Nov [cited 2019 Nov 3];136:3362-77. Available from: https://academic.oup.com/ brain/article-lookup/doi/10.1093/brain/awt265

30. Pawluski JL, Van Donkelaar E, Abrams Z, Houbart V, Fillet M, Steinbusch HWM, et al. Fluoxetine dose and administration method differentially affect hippocampal plasticity in adult female rats. Neural Plast 2014;2014.

31. Bianchi M, Shah AJ, Fone KCF, Atkins AR, Dawson LA, Heidbreder CA, et al. Fluoxetine administration modulates the cytoskeletal microtubular system in the rat hippocampus. Synapse. 2009 Apr [cited 2019 Nov 3];63:359-64. Available from: http:// doi.wiley.com/10.1002/syn.20614
32. Mateo S, Di Rienzo F, Reilly KT, Revol P, Delpuech C, Daligault $\mathrm{S}$, et al. Improvement of grasping after motor imagery in C6-C7 tetraplegia: A kinematic and MEG pilot study. Restor Neurol Neurosci 2015;33:543-55.

33. Metz GA, Whishaw IQ. The ladder rung walking task: A scoring system and its practical application. J Vis Exp 2009;

34. Jeffrey-Gauthier R, Josset N, Bretzner F, Leblond H. Facilitation of locomotor spinal networks activity by buspirone after a complete spinal cord lesion in mice. J Neurotrauma 2018;35:2208-21.

35. Kodama M, Fujioka T, Duman RS. Chronic olanzapine or fluoxetine administration increases cell proliferation in hippocampus and prefrontal cortex of adult rat. Biol Psychiatry 2004;56:570-80.

36. Kusakawa S, Nakamura K, Miyamoto Y, Sanbe A, Torii T, Yamauchi J, et al. Fluoxetine promotes gliogenesis during neural differentiation in mouse embryonic stem cells. J Neurosci Res 2010;88:3479-87.

37. Vetencourt JFM, Sale A, Viegi A, Baroncelli L, De Pasquale R, O'Leary OF, et al. The antidepressant fluoxetine restores plasticity in the adult visual cortex. Science (80- ). 2008;320:385-8.

38. Pleger B, Schwenkreis P, Grünberg C, Malin JP, Tegenthoff M. Fluoxetine facilitates use-dependent excitability of human primary motor cortex. Clin Neurophysiol 2004;115:2157-63.

39. Hajszan T, MacLusky NJ, Leranth C. Short-term treatment with the antidepressant fluoxetine triggers pyramidal dendritic spine synapse formation in rat hippocampus. Eur J Neurosci 2005;21:1299-303.

40. Murray KC, Stephens MJ, Ballou EW, Heckman CJ, Bennett DJ. Motoneuron excitability and muscle spasms are regulated by 5 HT2B and 5-HT2C receptor activity. J Neurophysiol 2011;105: 731-48.

41. Lee JY, Kang SR, Yune TY. Fluoxetine Prevents Oligodendrocyte Cell Death by Inhibiting Microglia Activation after Spinal Cord Injury. J Neurotrauma. 2015 May [cited 2020 Jan 13];32:633-44. Available from: http://www.liebertpub.com/doi/10.1089/neu.2014. 3527

42. Monaco CM, Gebhardt KM, Chlebowski SM, Shaw KE, Cheng JP, Henchir JJ, et al. A combined therapeutic regimen of buspirone and environmental enrichment is more efficacious than either alone in enhancing spatial learning in brain-injured pediatric rats. J Neurotrauma. 2014 Dec 1 [cited 2020 Sep 11];31:1934-41. Available from: /pmc/articles/PMC4238252/?report=abstract

43. Mello NK, Fivel PA, Kohut SJ. Effects of chronic buspirone treatment on nicotine and concurrent nicotine+cocaine self-administration. Neuropsychopharmacology [Internet]. 2013 Jun 21 [cited 2020 Sep 11];38:1264-75. Available from: www. neuropsychopharmacology.org

44. Bolin BL, Alcorn JL, Reynolds AR, Lile JA, Rush CR. Human drug discrimination: A primer and methodological review. Exp Clin Psychopharmacol [Internet]. 2016 Aug 1 [cited 2020 Sep 11];24:214-28. Available from:/pmc/articles/PMC4965187/ ?report=abstract

45. Shannonhouse JL, DuBois DW, Fincher AS, Vela AM, Henry MM, Wellman PJ, et al. Fluoxetine disrupts motivation and GABAergic signaling in adolescent female hamsters. Prog NeuroPsychopharmacology Biol Psychiatry 2016 Aug 1;69:19-30.

46. Adams S, Heckard D, Hassell J, Uphouse L. Factors influencing fluoxetine-induced sexual dysfunction in female rats. Behav Brain Res [Internet]. 2012 Nov 1 [cited 2020 Sep 11];235:73-81. Available from: /pmc/articles/PMC3432678/?report=abstract

47. Oh JE, Zupan B, Gross S, Toth M. Paradoxical anxiogenic response of juvenile mice to fluoxetine. Neuropsychopharmacology [Internet]. 2009 Sep 13 [cited 2020 Sep 11];34:2197-207. Available from: http://www.fda.gov/bbs/topics/

48. Freyvert Y, Yong NA, Morikawa E, Zdunowski S, Sarino ME, Gerasimenko Y, et al. Engaging cervical spinal circuitry with non-invasive spinal stimulation and buspirone to restore hand function in chronic motor complete patients. Sci Rep 2018 Dec 1;8. 
49. Bonin Pinto C, Morales-Quezada L, de Toledo Piza PV, Zeng D, Saleh Vélez FG, Ferreira IS, et al. Combining Fluoxetine and rTMS in Poststroke Motor Recovery: A Placebo-Controlled Double-Blind Randomized Phase 2 Clinical Trial. Neurorehabil Neural Repair [Internet]. 2019 Aug 1 [cited 2020 Sep 10];33:643-55. Available from: /pmc/articles/PMC6688938/?report=abstract

50. Edgerton VR, Roy RR, de Leon RD. Neural Darwinism in the Mammalian Spinal Cord. In: Spinal Cord Plasticity. Springer US; 2001. p. 185-206.

51. Bernshtein N. The co-ordination and regulation of movements. Oxford, New York: Pergamon Press; 1967. 196 p.

52. Bizzi E, Tresch MC, Saltiel P, d'Avella A. New perspectives on spinal motor systems. Nat Rev Neurosci 2000;1:101-8.

53. Wilson C, Simpson S, Van Emmerik R, Hamill J. Coordination variability and skill development in expert triple jumpers. Sport Biomech 2008;7:2-9.

54. Sawers A, Allen JL, Ting LH. Long-term training modifies the modular structure and organization of walking balance control. J Neurophysiol 2015 Dec 29;114:3359-73.

55. Jarvers C, Brosch T, Brechmann A, Woldeit ML, Schulz AL, Ohl FW, et al. Reversal learning in humans and gerbils: Dynamic control network facilitates learning. Front Neurosci 2016;

56. Wu HG, Miyamoto YR, Castro LNG, Ölveczky BP, Smith MA. Temporal structure of motor variability is dynamically regulated and predicts motor learning ability. Nat Neurosci 2014 Feb;17: 312-21.

57. Peroutka SJ, Sleight AJ, McCarthy BG, Pierce PA, Schmidt AW, Hekmatpanah CR. The clinical utility of pharmacological agents that act at serotonin receptors. Vol. 1, Journal of Neuropsychiatry and Clinical Neurosciences. 1989. p. 253-62.

58. Gobert A, Rivet JM, Cistarelli L, Melon C, Millan MJ. Buspirone modulates basal and fluoxetine-stimulated dialysate levels of dopamine, noradrenaline and serotonin in the frontal cortex of freely moving rats: Activation of serotonin $(1 \mathrm{~A})$ receptors and blockade of $\alpha 2$-adrenergic receptors underlie its actions. Neuroscience. 1999 Aug;93:1251-62.

59. Haleem DJ, Shireen E, Haleem MA. Somatodendritic and postsynaptic serotonin-1A receptors in the attenuation of haloperidol- induced catalepsy. Prog Neuro-Psychopharmacology Biol Psychiatry 2004 Dec;28:1323-9.

60. Huang YJ, Grau JW. Ionic plasticity and pain: The loss of descending serotonergic fibers after spinal cord injury transforms how GABA affects pain. Exp Neurol 2018 Aug 1;306:105-16.

61. Fink AJP, Croce KR, Huang ZJ, Abbott LF, Jessell TM, Azim E. Presynaptic inhibition of spinal sensory feedback ensures smooth movement. Nature. 2014;508:43-8.

62. G. Taccola, D. Sayenko, P. Gad, Y. Gerasimenko, V.R. Edgerton, (2018) And yet it moves : Recovery of volitional control after spinal cord injury. Progress in Neurobiology 160:64-81

63. Chia R, Zhong H, Vissel B, Edgerton VR, Gad P. Novel Activity Detection Algorithm to Characterize Spontaneous Stepping During Multimodal Spinal Neuromodulation After Mid-Thoracic Spinal Cord Injury in Rats. Front Syst Neurosci 2020 Jan 15;13.

64. De Leon RD, Hodgson JA, Roy RR, Edgerton VR. Locomotor capacity attributable to step training versus spontaneous recovery after spinalization in adult cats. J Neurophysiol 1998;79:1329-40.

65. Lynskey J V., Belanger A, Jung R. Activity-dependent plasticity in spinal cord injury. Vol. 45, Journal of Rehabilitation Research and Development. 2008. p. 229-40.

66. Dunlop SA. Activity-dependent plasticity: implications for recovery after spinal cord injury. Vol. 31, Trends in Neurosciences. 2008. p. $410-8$.

67. Dam M, Tonin P, De Boni A, Pizzolato G, Casson S, Ermani M, et al. Effects of fluoxetine and maprotiline on functional recovery in poststroke hemiplegic patients undergoing rehabilitation therapy. Stroke. 1996;27:1211-4.

68. Pariente J, Loubinoux I, Carel C, Albucher J-F, Leger A, Manelfe $\mathrm{C}$, et al. Fluoxetine modulates motor performance and cerebral activation of patients recovering from stroke. Ann Neurol. 2001 Dec 1 [cited 2020 Feb 8];50:718-29. Available from: http://doi.wiley. com/10.1002/ana.1257

Publisher's Note Springer Nature remains neutral with regard to jurisdictional claims in published maps and institutional affiliations. 